\title{
An adaptive retransmit mechanism for delay differentiated services in industrial WSNs
}

\author{
Ye Chen ${ }^{1}$, Wei Liu², Tian Wang ${ }^{3}$, Qingyong Deng ${ }^{4,5}$, Anfeng Liu ${ }^{1 *}$ (D) and Houbing Song ${ }^{6}$
}

\begin{abstract}
The Internet of Things (IoT) is the latest Internet development, with billions of Internet-connected devices and a wide range of industrial applications. Wireless sensor networks are an important part of the Internet of Things. It has received extensive attention from researchers due to its large-scale, self-organizing, and dynamic characteristics and has been widely used in industry, traffic information, military, environmental monitoring, and so on. With the development of microprocessor technology, sensor nodes are becoming more and more powerful, which enables the same wireless sensor networks (WSNs) platform to meet the different quality of service (QoS) requirements of many applications. Applications for industrial wireless sensor networks range from lower physical layers to higher application layers. The same wireless sensor network sometimes needs to process information from different layers. Traditional protocols lack differentiated services and cannot make full use of network resources. In this paper, an Adaptive Retransmit Mechanism for Delay Differentiated Services (ARM-DDS) scheme is proposed to meet different levels of delays of applications. Firstly, we analyze the impact of different retransmit mechanisms and parameter optimization on delays and energy consumption. Based on the results of the analysis, in ARM-DDS scheme, for routes with transmission delay tolerance, energy-saving retransmission mechanisms are used, and low-latency retransmission mechanisms are used for latency-sensitive routes. In this way, the data routing delays of different applications are guaranteed within bound and the energy consumption of the network is reduced. What is more, ARM-DDS scheme makes full use of the residual energy of the network and uses a small delay routing retransmit mechanism in the far-sink area to reduce end-to-end delay. Both theoretical analysis and simulation experiments show that under the premise of the same reliability requirements, ARM-DDS scheme reduces data transmission delay $12.1 \%$ and improves network energy utilization $28 \%$. Given that the reliability requirements of the data stream are different, the scheme can also extend the network lifetime.
\end{abstract}

Keywords: Industrial wireless sensor networks, Annular routing, Compressed sensing, Clustering, Energy utilization

\section{Introduction}

Wireless sensor networks (WSNs) are an important part of the Internet of Things (IoT). It has received extensive attention from researchers due to its large-scale, selforganizing, and dynamic characteristics and has been widely used in industry, traffic information, military, environmental monitoring, and so on. The development of modern industry is getting faster and faster, and the application research of industrial wireless sensor networks

\footnotetext{
* Correspondence: afengliu@mail.csu.edu.cn

'School of Computer Science and Engineering, Central South University, Changsha 410083, China

Full list of author information is available at the end of the article
}

is of great significance. With the development of microprocessor technology, sensing-based devices are becoming more and more powerful. The computing and storage capacity of smart sensing devices has been more than 10 years ago for personal computers [1-3]. The enrichment of sensing devices makes WSNs gradually evolve into a common sensing platform, which can provide data-aware services for a variety of applications [4-6]. Numerous applications can work together with the same WSNs as a platform to accomplish tasks at the same time, which greatly reduces the time and cost of application redeployment and operation of WSNs, and greatly promotes the development of WSNs [7-9]. For 
example, sensing nodes deployed on the roadside can monitor traffic flow, collect temperature data, and events can also be monitored, so that multiple different applications tasks can be performed on one platform $[10,11]$. This situation becomes more common as the processing capacity of sensor nodes increases, thus laying the foundation for the development of smart farming, smart transportation, smart industry, and smart cities [12-15].

Due to the diversity and complexity of applications running over WSNs [16], different applications may have different quality of service (QoS) requirements, especially for delay [13, 16-21]. For example, in the industrial automation production control line, the event data must be routed to the sink as soon as possible in the monitoring of fire and other dangerous events of important facilities and key equipment, otherwise, it will cause great loss of personnel and property. On the other hand, the humidity information monitoring is usually only needed to collect 3-4 times a day for the perception of environmental conditions such as the temperature of household crops, so the delay of data packet routing is tolerable and acceptable within a few minutes.

Another important issue in WSNs is the energy issue [11, 15, 20-24]. Because wireless sensor network nodes are powered by batteries and cannot be replaced in most scenarios, their energy is strictly limited $[19,25]$. Therefore, designing an energy-efficient routing strategy is an important issue in WSNs [16, 18, 20, 26, 27]. Another issue is to ensure the reliability of data transmission [28]. Because the wireless sensor network is based on wireless communication and data transmission to the sink often passes through multi-hop routing, its communication reliability is far lower than that of the wired network. According to relevant research, the link packet loss rate of the wireless sensor network is often as high as $10 \%$, in some cases even as high as $20-30 \%$ [28]. However, most applications have certain requirements for the reliability of data arriving at the sink. For example, in wireless communication with $90 \%$ reliability requirement, the reliability of data packet transmission after 10 hops routing is $34.9 \%$, while after 20 hops, the reliability is only $12.2 \%$. Therefore, in wireless communication, it is often necessary to take some reliability safeguard measures to make the reliability of data to the sink meet the threshold $\delta$. At the same time, different applications have different reliability thresholds, and some applications also tolerate the loss of some data packets, thus $\delta$ is usually a constant less than 1, such as $90 \%$ [28]. For example, in large-scale monitoring of crops, usually missing part of the data packets does not affect the effective monitoring of crops, because only a certain proportion of data can make the correct decision [28].
At present, most routing strategies are mainly aimed at a single application. Because the same application has simple QoS requirements, so a fixed QoS routing strategy can meet the requirements. However, with the enhancement of the function of sensing nodes, many applications can run on the same WSNs platform. At this time, it is very urgent to provide a routing strategy with differentiated services with QoS [16, 29]. In general, the strategy that makes routing delays smaller will consume more energy. Conversely, the routing strategies that consume less energy will have a high end-to-end delay $[21,26]$. Similarly, the reliability requirements of data transmission will also have an impact on energy consumption and delays. For example, wireless sensor networks usually use retransmitting mechanisms to ensure the reliability, so the higher the reliability requirements in applications [28], the greater the maximum number of retransmissions, the greater the delays caused by multiple retransmissions, and the more energy consumed, so the energy consumption and delays of applications with high-reliability requirements will be larger. Therefore, if applications with different QoS requirements are running on the same WSNs platform, if the same routing strategy is still adopted, that is, to select a routing strategy to satisfy the highest QoS requirements. Although this strategy can satisfy all applications' QoS requirements, the cost of the system is very high, which makes the overall lifetime of the system and the efficiency very low. Therefore, a strategy to provide differentiated services is urgently needed for the current WSNs.

There are already some routing strategies providing QoS differentiated services and studies on delay differentiated services also exist. Zhang et al. [16] proposed integrity and delay differentiated routing (IDDR) scheme to provide QoS differentiated services at a WSNs platform. In some applications, they are sensitive to data delays, while in some applications, they are sensitive to data integration requirements. Therefore, in the IDDR scheme, the delay-sensitive packets are routed to sink along the shortest path, thus minimizing delays. For packets sensitive to lose but not sensitive to delays, another routing strategy is adopted to avoid possible dropping on the hotspots in which with large data volume and high conflict and congestion. So, the IDDR scheme can guarantee two applications with different QoS requirements running on the same WSNs platform at the same time.

Obviously, for delay-sensitive data, although shortest routing can reduce delay, in the application of ultra-high delay requirement, the delay requirement cannot be satisfied even if the delay is reduced. That is to say, differentiated services are a best-effort strategy based on existing strategies, which has not been redesigned from 
the routing mechanism, so it can only satisfy a small range of delay differentiated services, so this mechanism is not applicable for applications that are particularly sensitive to delay requirements. On the other hand, in previous strategies, data that are not sensitive to delay will be bypassed. In fact, it is not advisable to increase the path length, because it will not bring any benefits to the system, but will only increase the delay and energy consumption, especially in data-sparse networks. Given the above problems, an Adaptive Retransmit Mechanism for Delay Differentiated Services (ARM-DDS) scheme is proposed to meet different levels of delays of applications; the main innovations of the scheme are as follows:

(1) An Adaptive Retransmit Mechanism for Delay Differentiated Services (ARM-DDS) scheme is proposed to meet the needs of applications with delay differentiated services. Firstly, we analyze the retransmit mechanism in depth and get the influence of different to retransmit modes and retransmit times on delay and energy consumption, as well as the optimal selection of retransmitting parameters on the premise of ensuring certain reliability of data transmission. In the ARM-DDS scheme, for routes with transmission delay tolerance, energy-saving retransmission mechanisms are used, and for latency-sensitive routes, low-latency retransmission mechanisms are used. In this way, the data routing delays of different applications are guaranteed within the bound and the energy consumption of the network is reduced, so delay differentiated services are realized very well.

(2) What is more, the ARM-DDS scheme makes full use of the residual energy of the network and uses a small delay routing retransmit mechanism in the far-sink area to reduce end-to-end delay. Therefore, the ARM-DDS scheme can further reduce the delay based on improving energy utilization.

(3) Both theoretical analysis and simulation results show that, compared with previous studies, the ARM-DDS scheme has the following advantages:

(a) ARM-DDS can meet the delay requirements in a wide range. In previous studies, the delay range allowed by the delay differentiated services scheme is relatively narrow and cannot meet the highly sensitive requirements of different applications for delay, while the ARM-DDS scheme can reduce the delay to one third of the existing strategies, so it has a strong practical significance and a wide range of adaptability. (b) Compared with the highest QoS strategy, the ARM-DDS scheme can greatly reduce energy consumption and prolong network lifetime.

(c) Compared with the general stop-wait automatic retransmit protocol, the ARM-DDS scheme reduces data transmission delay by $12.1 \%$ and improves network energy utilization by $28 \%$.

The rest of this paper is organized as follows: Section 2 shows the methods. Section 3 reviews related works. Section 4 describes the network model and problem statements. In Section 5, the detailed design of the ARM-DDS scheme is presented. The results of the theoretical performance analysis are given in Section 6. The experiment results are given in Section 7. The conclusion is presented in Section 8. The list of abbreviations is in the "Abbreviations" section.

\section{Methods}

In WSN, transmission of data with different delay requirements and reliability requirements is common, so it is important to differentiate services. Therefore, a method of differentiated services to improve the utilization of network resources is proposed. Differentiated services can effectively improve energy-efficiency. Because traditional routing algorithms cannot perform differentiated services for different requirements of data, transmission methods that use the highest latency requirements and reliability requirements for all data waste energy. The use of differentiated services for data with different delay requirements can reduce the amount of data sent by low-latency sensitivity and reduce the energy consumption of the network. Some data have highlatency-sensitivity requirements and can utilize the residual energy of the non-hotspot region to employ a lower latency retransmission mechanism. Therefore, such a differentiation method can meet the delay requirements of all data and improve energy utilization without affecting the life of the network. On the other hand, differentiated services for data streams with different reliability requirements can extend network lifetime, because packet reliability is guaranteed by the retransmission mechanism. Therefore, the data packet of different reliability requirements is differentiated, and the number of retransmissions of the data packet required by the low reliability is reduced, that is, the number of data retransmissions of the network is reduced, and the network life is prolonged. Therefore, the differentiated service method can meet the data transmission requirements of higher delay sensitivity, improve energy utilization, and extend network lifetime. The background and related work on this method can be found in Section 3.

\section{Background and related work}

More and more wireless sensing devices are currently being applied in a variety of fields [30, 31], such as military, industrial, agricultural, and daily life [32,33]. And the number of these sensing devices is huge. According 
to [1], the number of devices currently connected to the Internet has exceeded that of humans, thus greatly promoting the development of the Internet of Things (IoT) $[1,3,5,11,32,34]$. These sensing devices have huge computing and storage resources, which have caused major changes in the current network structure $[1,11$, $32,34,35]$, mainly reflected in edge computing. Fog computing has become the focus of current research [1, 11, 32, 34, 35]. These new network structures are combined with the emerging artificial intelligence technology [36, 37], which brings unprecedented opportunities for the Internet of Things (IoT) and greatly promotes network development $[34,35]$. As wireless sensor networks (WSNs) is the most important component of IoT, more and more applications are deployed in the same WSNs, which leads the research of WSNs to a new stage [17, 19], from single research to hybrid and differentiated services.

The most important task of WSNs is to perceive and acquire information in the surrounding environment and pass the perceived data to the sink for corresponding processing $[13,17,20]$. Since different applications have different delay requirements, the range involved in the delay is very wide, involving from the MAC layer, the network layer, to the application layer [13, 17-21, 26]. And delay is closely related to other related performance indicators, such as a retransmission mechanism designed due to the unreliability of network transmission. The retransmission mechanism will increase the delay, and also increase the energy consumption of the node. The reliability of the communication link is related to the transmission power of the node. Increasing the transmission power of the node can improve the reliability of the communication link, thereby reducing the retransmission of the data packet, reducing the delay, and reducing the extra energy consumption caused by the retransmission. However, increasing the transmission power of a node itself increases the energy consumption of the node. These, in turn, interact with the duty cycle working mode adopted by the node, routing mechanisms such as multi-path routing, and coding methods of the application layer. Therefore, the optimization of wireless sensor networks is a very complicated challenging issue. This section will discuss the related work.

\subsection{Retransmission protocols and mechanisms}

The retransmission protocol is an important mechanism to ensure the reliability of wireless transmission and is one of the most important research contents in WSNs. As mentioned earlier, the communication quality of WSNs is often very different due to the communication distance between nodes, transmission power, and the surrounding environment. In some networks, the packet loss rate is as high as $20-23 \%$, so some reliability measures are needed to ensure the reliability of packet transmission. At the same time, the strategy of guaranteeing the reliability of data transmission often affects the delay of data transmission. Therefore, the strategy of ensuring the reliability of data transmission is considered simultaneously with the delay optimization strategy.

(1) Send-and-wait automatic repeat-request (SWARQ) protocols. The SW-ARQ protocol is a basic data transmission protocol that guarantees the reliability of wireless communication [38]. It mainly guarantees the reliability of data transmission through the retransmission mechanism. Its main operating mechanism is as follows: Sender starts to send packets after establishing a connection with the receiver, and the receiver returns an acknowledge (ACK) to the sender after receiving the packet. After receiving the ACK, sender knows that the data packet has been successfully received by the receiver, so it can start the transmission of the next packet. If the packet sent by the sender is lost during the transmission, the receiver will not return an ACK back because of not having received the packet [38]. Thus, after the sender waits for a timeout period and does not receive the ACK, it considers that the transmitted data packet has been lost, and thereby retransmits the data packet. The sending and receiving process is the same as that of the first sending of the data packet. From the above process, the reliability of the SW-ARQ protocol data transmission is guaranteed by retransmitting the data packet multiple times. Let the probability of successful data transmission be $p_{i}^{s}$, then $n$ data retransmissions can probabilistically guarantee the probability of successful transmission is $1-\left(1-p_{i}^{s}\right)^{n}$. It can be seen that multiple retransmission mechanisms can significantly improve the reliability of data transmission but will increase the delay of data transmission. In data communication, the time it takes for the sender to send a packet to receive an ACK is called round-trip time (RTT), and the sender takes longer to perform a retransmission. It needs to wait for a time to out (RTO) time before starting a retransmission, while the RTO duration is longer than the RTT, because delay is the accumulation of time taken by multiple retransmissions. Thus, the more data retransmissions, the greater the delay [38]. In order to control the delay within the allowable range, it is often necessary to set a maximum number of retransmissions $m$. That is, when the maximum number of retransmissions of the same packet reaches $m$, the packet is not retransmitted, but discarded. It is easy to get the maximum number of retransmissions: Since the reliability of data transmission for $m$ times is required to be $1-\left(1-p_{i}^{s}\right)^{m}>\delta$, the reliability of data transmission can be guaranteed to meet the requirement when $m$ 
$=\left\lceil\lg (1-\delta) / \lg \left(1-p_{i}^{s}\right)\right\rceil$. Setting the maximum number of retransmissions prevents extreme situations during a packet transmission: always unsuccessful transmission. Of course, in general, the packet transmission success rate $p_{i}^{s}$ is relatively high, which is $80 \%$ or more. Therefore, in practice, the value of the data transmission success rate $p_{i}^{s}$ is relatively high. Therefore, although the maximum number of retransmissions $m$ is set, the average number of successful data transmissions is not large in the case where $\delta$ is less than 1 , much smaller than $m$ [38].

The above retransmission mechanism is mainly aimed at the reliability transmission of hop to hop. According to source node routing and multi-hop routing of the data packet, the above retransmission mechanism also has control strategies divided into end to end (E2E) and hop by hop (HBH) [38]. The control mode of E2E is that the source node sends the data packet to the sink; then, the latter returns the ACK after receiving the data packet. In this control mode, once the source node receives the ACK, it knows that the data packet has been successfully received by the sink, and thus begins to send the next packet. However, in this mode, the data packet has to go through multiple hops to reach the sink. Assuming that the hop count of the data packet from the source node to the sink is $h$, the success rate of the packet to the sink through $k$ hops' routing is $p^{h}$. Obviously, if the success rate of one hop of the ACK when it returns is $\beta$, the probability that the ACK successfully returns to the source node is $\beta^{h}$. Therefore, the probability that the source node successfully receives the ACK returned is $p^{h} \cdot \beta^{h}$. If $\rho=90 \%, \beta=95 \%$, and $h=10$, the probability that the source node can receive the ACK after sending the data packet $<21 \%$. To make the reliability rate of the data successfully reaching the sink, $\delta=$ $90 \%$, the source node needs to send 10 times on average to guarantee the rate. For each retransmission, the packet needs to go through 10 hops to reach the sink, and the ACK returned by the sink needs to go through 10 hops to return to the source node, so the elapsed time (delay) is very long, and the RTO time of a retransmission will be longer. This shows that in the E2E control mode, the number of retransmissions of the source node is large, and the delay is large too [38]. However, this control method has one advantage that has not been noticed in previous studies: In an ideal wireless sensor network with high reliability of data transmission (the transmission reliability rate can be considered as $100 \%$ ), since sink is the end point of data transmission of all nodes, the nodes that are close to the sink area bear a large amount of data, which is called hotspots. Due to the hotspots, the energy consumption of nodes near the sink area is much higher than in other areas, which may lead to early network death. This situation seriously damages the lifetime of the network. In the reliability control mode of E2E, since part of the data packets sent by the source node will gradually lose during their transmission to the sink, which will reduce the impact of hotspots. Hop by hop (HBP) reliability control is a hopby-hop reliability control method [38]. In this way, if the reliability of receiving the data packet by the sink is $\delta$, and the data packet needs to go through $h$ hops to reach the sink, and when the data transmission reliability of each hop is $\sqrt[h]{\delta}$, the reliability after the $h$-hop routing of the data packet is guaranteed to be $\delta$. In the HBP mode, to require the reliability of each hop to be $\sqrt[h]{\delta}$, that is, to require this process, the sender sends the data packets to the receiver, then receiver returns the ACK to the sender after having received, but if the sender does not receive the ACK, timeout retransmission is implemented. If the sender sets its maximum number of retransmissions $m=\left\lceil\lg (1-\sqrt[h]{\delta}) / \lg \left(1-p_{i}^{s}\right)\right\rceil$, the end to end reliability of data transmission will be guaranteed to be $\delta$. In this HBP control mode, the control mode adopted by each node is single-hop control, and thus its end to end delay is the delay accumulation of each hop. Relatively speaking, the nodes of the HBP mode bear less data.

It can be seen from the above SW-ARQ mechanism that the biggest feature of this retransmission mechanism is that the protocol is simple and easy to implement [38]. Moreover, in this way, the extra energy consumption of the node is due to the energy consumption by transmitting the ACK, and the length of the ACK is relatively smaller than the length of the data packet, so the additional energy consumption is relatively small, and thus the energy consumption of the protocol is relatively small compared to the mechanism introduced later. However, the biggest shortcoming of this method is that each retransmission generates a timeout period, and the data packets may be needed to be retransmitted multiple times, resulting in a larger delay for this protocol.

In order to reduce delay and the large deficit of the SW-ARQ protocol, the researchers proposed an improved protocol for SW-ARQ. Go-Back-N protocol (GBN) [39] is just one of the improved protocols. The main operation of the GBN protocol is the following: The sender is no longer a serial mode that it sends a packet at a time and waits for an ACK to return like the SW-ARQ protocol [39]. In the GBN protocol, the sender continuously sends multiple data packets, and the data packet contains the sequence number of itself. If the receiver finds that the sequence number of the received packet is not continuous, which indicates that there is a packet loss during the transmission, therefore, the receiver sends a NACK to the sender, indicating the smallest sequence number of the packet in the unreceived 
packets. After the sender receives the NACK, it retransmits from the sequence number of the packet indicated in the NACK. The GBN protocol speeds up the transmission of data packets, thereby reducing delay [39].

Selective repeat protocol (SR) [40] is an improvement to the GBN protocol. In this protocol, the sender sends the packet in a similar manner to the GBN protocol. However, when the receiver returns the NACK of the unreceived packets, the SR protocol only retransmits the packets that were not successfully transmitted, thus reducing the system overhead.

The delay of the retransmission protocol mainly comes from the retransmission of the data packet, and the retransmission of the data packet will inevitably go through a TTO time, so the time taken is long. Multiple retransmissions and multi-hop routing will make the end to end delay very large. Therefore, if using the mechanism that does not return ACK, the delay can be reduced. Therefore, some researchers have proposed the mechanism of $s$ packet rerouting. In this mechanism, the sender repeatedly sends the same data packet $m$ times, and the $s$ data packets all have a certain probability of reaching the sink. Therefore, after repeated transmissions for $m$ times, the value of data transmission reliability can be ensured to meet the application requirements $\delta$. Compared with the SW-ARQ protocol, since the receiver does not need to return ACK in this protocol, the delay is the least, but the node has the highest energy consumption. As previously analyzed, in the SW-ARQ protocol, the average expected number of times that a sender sends a packet is much smaller than the maximum number of retransmissions $m$. However, in the mechanism without feedback, the number of times the data packet is sent by each node is the maximum number of retransmissions $m$. Therefore, the energy consumption of this protocol is very large and rarely used in wireless sensor networks.

\subsection{Routing and delay optimization work}

(1) Multipath routing mechanism. The proposed multipath routing mechanism is not proposed for communication links and unreliability but it is similar to the above non-feedback communication protocol. The multi-path routing mechanism is that the sender routes the data packets from multiple routes to the sink at the same time. Therefore, as long as the data packets of one route can be successfully routed, the data packet transmission can be successful [41]. Multi-path routing mechanism is often proposed to defend against security attacks, so that if a route is attacked, there are still other routes that can successfully reach the sink [42]. Corresponding to this is the Security and Energy-efficient Disjoint
Route (SEDR) scheme. SEDR scheme using $(T, M)$ threshold secret-sharing mechanism divides the data into $M$ shares, which route to the sink along different routes. If sink receives the $T$ shares of the $M$ shares, the sink can get the information of the entire packet [43]. And the attacker cannot get the packet information if the number of shares it gets is less than $T$. (1) Although this method is designed for security, it plays a role in improving the reliability of data transmission.

Another strategy to improve the reliability of data transmission is the packet reproduction method [44]. In such a routing strategy, since some data packets are gradually lost in the routing process, in the packet reproduction routing method, the packet is reproduced once every certain number of hops (such as $i$ hops) [44], that is, to copy the source packet to $M$ shares, each of which is transmitted forward along a different routing path. Some of the $M$ packets in the process of routing will disappear. In order to ensure that there are packets that can reach the sink in the end, when each data packet is routed $i$ hops, reproducing $M$ shares for rerouting from different routes to make up for those lost packets in the routing process. Thus, the data packet can be guaranteed to reach the sink with high reliability. This packet reproduction method has a lower cost than the multi-path routing, and the reliability of the data routing is high [44].

(2) Opportunistic routing. Opportunistic routing is also an effective routing method for reducing delay. The essence of this method is to make full use of the broadcast characteristics of wireless communication, so that the delay of routing can be made smaller on the basis of ensuring the reliability of data transmission. Specifically, the method used by opportunistic routing is the following: Due to the unreliability of data transmission, the sender will select multiple receivers for transmission in opportunistic routing in order to improve the reliability of data routing. Since multiple receivers are selected for one data transmission, assuming the transmission reliability is $p$, when $n$ receivers are selected in the opportunistic routing, the probability that the sender successfully transmits the data after the calculation is $1-(1-p)^{n}$. It can be seen that a data transmission once of opportunistic routing is equivalent to $c$ times of transmissions in the SW-ARQ protocol, so that the delay can be effectively reduced. But in this method, you need to select $n$ receivers at the same time. Such wireless sensor nodes can easily select $n$ receivers in a non-duty cycle network, however, which is difficult in the duty cycle 
based WSNs, due to the periodic awake/sleep of the nodes.

(3) Broadcast routing method. To overcome the packet loss of data transmission and reduce delay, Joo et al. [45] proposed a broadcast data transmission method in data fusion network. In some data fusions, $n$ multiple data packets can be merged into one data packet. For example, in a wireless sensor network, the average temperature and average humidity in the surrounding environment are monitored. When multiple monitoring data meet, the average value can be calculated. The node only needs to transmit the average value when transmitting the data value. In the network of packets loss, the data packet has a certain loss ratio in the process of routing to the sink. Therefore, the method adopted by Joo et al. [46] is that each node that receives the data routes to the sink in a broadcast manner. By adopting such a method, the reliability of the data routing can be significantly improved. Because, after a node broadcasts, the data packet received by nodes that are closer to the sink than the broadcast node, is merged with its original data, which continues to be broadcasted forward. Therefore, if there are $m$ nodes in the broadcast range of the original node, it can broadcast forward again, the probability of successful data transmission is $1-\left(1-p_{i}^{s}\right)^{m}$. Each node that successfully receives the data packet will broadcast forward in the same way, so the probability of success of each route is $1-\left(1-p_{i}^{s}\right)^{m}$. This greatly increases the probability of data transmission to the sink. Also, in this way, the delay is relatively small. Because only the packets that have been successfully transmitted survive and are continuously routed forward, this is equivalent to a multi-path routing strategy, and the time used by the packet first arriving in the sink is the transmission delay of this packet.

\subsection{Transmit power and delay optimization work}

In a network with high reliability of the communication link, the data packet does not need to be retransmitted, so its delay is small. While in a network with low reliability of the communication link, the data needs to be retransmitted multiple times, and the time consumed by one retransmission far exceeds the time of the first data transmission. Therefore, the method of improving the reliability of the communication link can be used to reduce delay and improve data transmission reliability. Thus, there are also some methods to reduce delay and improve data transmission reliability by improving the quality of communication links [46]. One of the more effective methods is to improve the data transmission power. The central idea of this approach is that the communication success rate between nodes in a wireless router network is not only related to the distance between the nodes but also related to the transmit power of the sender nodes. The higher the transmit power of the sender nodes, the higher the signal-to-noise ratio (SNR) of the received signal of the receiver and the higher the success rate of receiving data [46]. It can be seen from the foregoing discussion that if the data transmission success rate is higher, the number of retransmissions required by the node is smaller, so that the delay caused by the retransmission can be effectively reduced. For example, in a network with a data transmission success rate of $98 \%$, the probability of successfully reaching the sink after 10 hops is $81.7 \%$. While in a network with a data transmission success rate of $80 \%$, the success rate of the packet reaching the sink after a 10-hop routing is only $10.7 \%$. It can be seen that increasing the success rate of data transmission can significantly reduce the number of retransmissions thus reducing delay [46]. Based on the above ideas, some researchers have proposed the strategy of increasing the transmission power of the sender to improve the reliability of data transmission, thus to reduce the delay. But the real strategy of improving the node's transmit power is more complicated. Because increasing the transmit power of a node requires more energy consumption, but the energy of the node is very limited. Thus, to increase the transmit power of the node, it should minimize the energy consumption of data per bit successfully transmitted [46]. The sender's transmit power is a nonlinear relationship with the successful transmission of the packet (see Fig. 1). If the communication distance between the nodes is fixed, increasing the transmit power of the sender can increase the receiver's reception rate. However, after the receiver's receiving rate reaches a certain value, it rises very little if the sender's power continues to be increased. Therefore, when the transmit power of the sender can be adjusted to the optimized value, the energy consumption for successfully transmitting each bit of data can be minimized. In terms of reducing the delay, increasing the sending power of the sender helps to reduce the number of retransmissions, thereby reducing delay [46].

\subsection{Differentiated service-related work}

Due to the development of current microprocessors, multiple applications can run in the same WSNs at the same time. Because different applications require different QoS indicators, research on differentiated services has only just begun, but there have been some researches about this. 


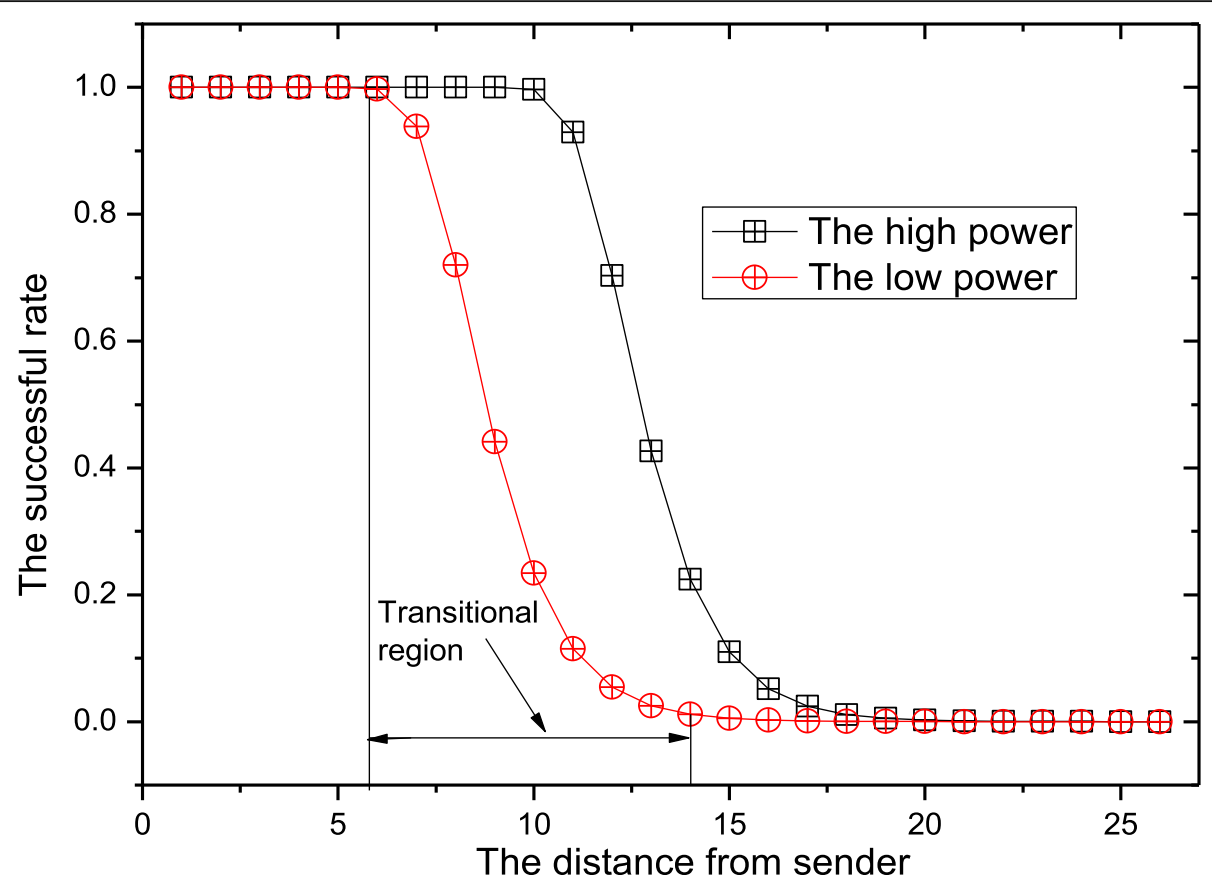

Fig. 1 The relationship between the successful rate of the packets being received and power

Zhang et al. [16] proposed an integrity and delay differentiated routing (IDDR) scheme, which is such a differentiating service strategy. In IDDR scheme, there are two different types of network applications. One is data integrity, which is an application of packet loss sensitivity, requiring an extremely low packet loss ratio. The other type is an application of delay sensitivity, which has high requirements for delay. In WSNs, the shortest routing method is generally adopted, which causes hotspots near the sink area. The hotspot area bears a large amount of data, which on the one hand makes the energy consumption of nodes in this area large. On the other hand, congestion occurs in the hotspots area, due to the large amount of data transmitted by the nodes in this area. The result of congestion is high packet loss rate and large data routing delay. Therefore, the solution adopted by the IDDR scheme is using the shortest routing method for delay-sensitive packets to reduce their delay. For data packets with high data integrity requirements, a detour is used. By bypassing the hotspots area, although the length of the route is increased, the congestion is small, thus meeting the requirements of data integrity. Therefore, IDDR can differentiate services.

Huang et al. [47] proposed a very effective delay in differentiated services (DDS) for data fusion networks. In a data fusion network, if a packet waits for a period of time for more packets on the intermediate node of the route, the amount of data reduced will be larger after more data packets are merged, thereby reducing the energy consumption of the route and improving the network life. Therefore, from the perspective of reducing energy consumption, the longer the data packet stays on the node, the more data fusion can be performed, which can reduce energy consumption and thus help to improve network life. However, the longer the packet stays on the node, the greater the delay of the packet reaching the sink, which affects its usability. Thus, the method adopted by Huang et al. [47] is the following: For data with high delay requirements, set a smaller dwell time threshold to speed up data routing and reduce delay, and for data with less demanding delay, set a larger dwell time threshold to make more data packets meet for data fusion thus reducing energy consumption and improving network life.

\section{The system model and problem statement}

\subsection{The network model}

In this paper, a system model of a classical planar wireless sensor network is used. The system model is referenced to [48]. The system model structure is as follows:

(1) The research environment of this paper is a planar wireless sensor network, which is a circular network model with $R$ as the radius of the network and $r$ as the radius of each hop. There are many sensor nodes and one sink node in the planar network. Among them, the sensor nodes have the same initial energy. The sensor node cannot be replaced in the middle of use. For the sink node, there is no energy limitation $[17,18,26]$. The sensor nodes are 
evenly distributed according to the density $\rho$ in the network centered on the sink node. The power of the node can be adjusted according to the distance of the transmitted data [49]. In a transmission cycle, each sensor node collects data and generates a packet. The packet will be transmitted to the sink via the set multi-hop route.

(2) In the wireless sensor network studied in this paper, the sensor node position is fixed. And when the sensor node transmits data, its transmission path does not change. Data routing uses the shortest path by the policy. Since the probability distribution of network events, in reality, is relatively sparse, this paper does not consider the congestion phenomenon on the nodes, that is, the queue delay.

(3) When the node performs data transmission, the success rate of node $i$ to transmit data to the next hop is $p_{i}^{s}$, and the success rate of the ACK returned after the next hop receives the data is $q_{i}^{a}$. If the transmit failed, it will be retransmitted.

\subsection{System parameters}

The local energy model used in this paper is referred to [50]. Let $\varpi_{s}$ represent the energy consumption of data transmission, as shown in Eq. (1).The energy consumption $\varpi_{\text {rec }}$ of the received data is given by Eq. (2).

$$
\begin{aligned}
& \begin{cases}\varpi_{s}=\alpha \ell_{s} s^{2}+\alpha E_{\text {elec }} & \text { if } s<s_{t d} \\
\varpi_{s}=\alpha \ell_{m} s^{4}+\alpha E_{\text {elec }} & \text { if } s>s_{t d}\end{cases} \\
& \varpi_{\text {rec }}=\alpha E_{\text {elec }}
\end{aligned}
$$

Among them, the transmission circuit loss is represented by $E_{\text {elec }}$. The energy consumption of data transmission uses the free space $\left(s^{2}\right.$ power loss) and multipath fading ( $s^{4}$ power loss) channel model according to whether the distance exceeds the threshold $s_{t d}$. When the power amplifier loss is based on the free space model, $\ell_{s}$ is used instead; when the transmission distance is greater than or equal to the threshold $s_{t d}, \ell_{m}$ is selected as the power amplifier loss of the multi-antenna model. $\alpha$ is the number of bits in the data packet. The above parameter settings are shown in Table 1 [50]. The system parameters adopted in this paper are listed in Table 2.

Table 1 Network parameters

\begin{tabular}{ll}
\hline Parameter & Value \\
\hline Threshold distance $\left(r_{0}\right)(\mathrm{m})$ & 87 \\
Sensing range $(r)(\mathrm{m})$ & $\leq 80$ \\
Eelec $(\mathrm{nJ} / \mathrm{bit})$ & 50 \\
$\varepsilon_{f \mathrm{~s}}\left(\mathrm{pJ} / \mathrm{bit} / \mathrm{m}^{2}\right)$ & 10 \\
$\varepsilon_{\text {amp }}\left(\mathrm{pJ} / \mathrm{bit} / \mathrm{m}^{4}\right)$ & 0.0013 \\
Initial energy $\left(E_{\text {init }}\right)(\mathrm{J})$ & 0.5 \\
\hline
\end{tabular}

\subsection{Problem statement}

The main goal of this paper is to design a communication approach to reduce the transmission delay of WSNs by improving the network energy utilization, and to ensure that the increase of Adaptive Retransmit Mechanism for Delay Differentiated Services (ARM-DDS) protocol.

The problem of the ARM-DDS protocol can be described as differentiated services for different transmission delay requirements, to meet the broad delay requirements. Data packets with different delay requirements and reliability requirements are processed differently to optimize the delay and energy utilization of the entire network. WSN data collection routing can be characterized by several performance indicators, as described below:

(1) End-to-end delay (denoted as $\mathcal{Y}_{\text {ETE}}$ ) refers to the time from when the source node sends a packet to the sink. After the source node sends the data packet, it will reach the sink through several relay nodes. Each time a packet is forwarded via a relay node, it takes time. Therefore, the end-to-end delay is the sum of the transmission delays per hop. The end-to-end delay minimization can be expressed as follows:

$$
\min \left(\mathcal{Y}_{\mathrm{ETE}}\right)=\min \left(\sum y_{i}\right)
$$

(2) The effective energy utilization rate (expressed as $\eta$ ) refers to the ratio of the energy used in the network to the total energy of the network. Considering that $o_{i}$ is the energy consumption of node $i$ in the network, $\mathcal{O}_{i}$ is the initial energy of the node, so the maximum effective energy utilization is as follows:

$$
\max (\eta)=\max \left(\frac{\sum o_{i}}{\sum \mathcal{O}_{i}}\right)
$$

(3) Definition of network lifetime (expressed as $\mathcal{L}$ ) [1, $12,14]$. Network lifetime is defined as the death time of the first node in the network. $\mathcal{O}_{i}$ is the energy consumption of node $i$, and $\mathcal{O}_{i}$ is the initial energy of each node. Therefore, the maximum network lifetime is as follows: 
Table 2 System parameters

\begin{tabular}{lll}
\hline Parameter & Value & Description \\
\hline$p_{i}^{s}$ & $60 \%-95 \%$ & Probability of data being successfully sent to the subsequent node \\
$q_{i}^{a}$ & $80 \%-95 \%$ & Probability of ACK successful transmission \\
$\delta$ & $90 \%-100 \%$ & End-to-end reliability of data packets \\
$\tau_{\text {round }}$ & $50 \mathrm{~ms}$ & round-trip time \\
$\tau_{\text {rtto }}$ & $60 \mathrm{~ms}$ & round-trip time to out \\
$s$ & $10 \mathrm{~ms}$ & The minimum interval time of next data packets being sent \\
$b$ & $10 \mathrm{~ms}$ & The minimum interval time of next ACK being sent \\
$\mu$ & $10 \mathrm{~ms}$ & Timeout interval of retransmission \\
\hline
\end{tabular}

$$
\max (\mathcal{L})=\max \left(\mathcal{O}_{i} / o_{i}\right)
$$

In summary, the research questions in this paper can be summarized as follows. And the parameters related to the calculation are shown in Table 3.

$$
\left\{\begin{array}{c}
\min \left(\mathcal{Y}_{\mathrm{ETE}}\right)=\min \left(\sum y_{i}\right) \\
\max (\eta)=\max \left(\frac{\sum o_{i}}{\sum \mathcal{O}_{i}}\right) \\
\max (\mathcal{L})=\max \left(\frac{O_{i}}{o_{i}}\right) \\
\text { s.t. } \delta_{i}^{e 2 e}=\prod_{k \in \text { path to sink of node } i^{\lambda^{k} \geq \delta}}
\end{array}\right.
$$

\section{ARM-DDS strategy}

\subsection{Research motivation}

The Adaptive Retransmit Mechanism for Delay Differentiated Services (ARM-DDS) scheme is mainly proposed for the research problem in which no effective methods work on the distinction of delay differentiated service. The main goal of ARM-DDS is to reduce the energy consumption of nodes as much as possible. Under the premise of ensuring the reliability of data transmission to meet the application requirements, it can provide a wide range of differentiated services for the delay. The research motivation of this paper mainly comes from the following points.

(1) This paper provides a wide range of adaptive retransmission mechanism for delay differentiated services. The previous SW-ARQ protocol, GBN protocol, and SR protocol are all best-effort protocols, that is, the protocol takes into the optimized operation of both energy consumption and delay. Therefore, this kind of protocol uses the same service mechanism for the applications, so it cannot work on the situation of delay differentiated services in the development of wireless sensor networks. We have found that in the original retransmission mechanism, the same rules of sending/confirming data are working on the same packets. For example, in SW-ARQ, after sending a data packet, it waits for the ACK confirmation message (see Fig. 1). However, in the GBN protocol, the retransmission

Table 3 Parameters related to the calculation

\begin{tabular}{ll}
\hline Notation & Description \\
\hline$c$ & Persistent transmission times for the first time \\
$n$ & Number of total nodes in network \\
$\gamma_{h}(\delta)$ & The maximum number of retransmissions of hop $h$ \\
$e_{p}^{t}, e_{p}^{r}$ & Energy consumption of node for receiving and sending a data packet respectively \\
$e_{A}^{t}, e_{A}^{r}$ & Energy consumption of node for receiving and sending an ACK respectively \\
$\omega_{i}$ & Data E2E delay of node $i$ in ARM-DDS protocol \\
$D_{\xi, i}^{k, t}, X_{\xi, i}^{k, t}$ & Number of node $i$ for sending and receiving data packets in SW-ARQ protocol respectively \\
$W_{i}^{t}, W_{i}^{r}$ & Network delay \\
$S_{h}^{\text {send }, S_{h}^{r e c}}$ & Number of hop $h$ for sending and receiving data packets in ARM-DDS protocol respectively \\
$S_{h}^{A S}, S_{h}^{A R}$ & Number of node $i$ for sending and receiving data packets in ARM-DDS protocol respectively \\
$k$ & Data stream number, indicating different data streams \\
\multicolumn{1}{c|}{$\Delta$} & A constant less than the radius \\
\hline
\end{tabular}


orders depend on the NACK whether to confirm the message receiving after sending data packet. In the SR protocol, the loss packet can be directly retransmitted after receiving the NACK. To further reduce the delay, we use the methods in this paper: for the perfect application system of delay, when the first packet is sent, it is repeatedly sent $c$ times (see Fig. 2). When $c=1$, it is the same as the retransmission mechanism currently used. When $c>$ 1 , the delay can be reduced. The reason is this, when $c>1$, since the continuously sent $c$ times, the probability that the port receives the data packet is greatly improved, that is, the original $p_{i}^{s}$ is reduced to $1-\left(1-p_{i}^{s}\right)^{c}$. Therefore, the subsequent retransmission can be effectively reduced. The probability of being able to effectively reduce the delay. The delay distinguishing service can be obtained by adjusting the parameter $c$. When $c=1$, it is the retransmission mechanism that has been proposed. When $c$ selects different values, the delay of data transmission will be different. Generally, when the $c$ value is small, increasing the value of $c$ will reduce the delay. However, taking the largest possible $c$ value does not achieve the goal of reducing the delay. When the packet is sent with a minimum interval of $s$, it takes at least $(c-1) s$ to send $c$ packets. If the value of $c$ is too large, the delay will also increase. Therefore, the delay of the repeated transmission $c$ is large, and the delay is limited. On the one hand, the data packet is sent $c$ times at a time, and its energy consumption increases linearly with the increase of $c$. Therefore, the tradeoff between delay and energy consumption is needed to obtain an optimized $c$ value. As can be seen from the above analysis, The retransmission mechanism proposed in this paper can obtain different delay levels by selecting the difference of the parameter $c$. Overcoming the previous strategy, it only provides a delay level, which makes the ARM-DDS proposed in this paper have a wide delay service.

(2) Based on the first point, this paper proposes a service-differentiating architecture. This architecture is: For applications with high delay requirements, a large retransmission mechanism under $c$ value is used to make the data transmission delay small. For applications with low delay requirements, a retransmission mechanism with a smaller $c$ value is used. Since the energy consumption of the node is proportional to the value of $c$, the delay is inversely proportional to the value of $\mathrm{c}$. Thus, to reduce the delay system requires more energy consumption. However, the ARM-DDS strategy provides better overall performance than the previous strategy because of the differentiated services provided. The main reasons are as follows: (a) The SW-ARQ protocol lacks differentiated services. In order to meet QoS requirements of all the data when using the SW-ARQ protocol, only the strategy that meets the highest QoS requirements in the network can be adopted. Therefore, comprehensive energy consumption is much higher than the strategy proposed in this paper. (b) There is no differentiation strategy proposed in this paper. The previous strategy is a kind of pseudo-differentiated service, rather than truly differentiated services, because, in those strategies, the strategy can only reach one service level. The retransmission

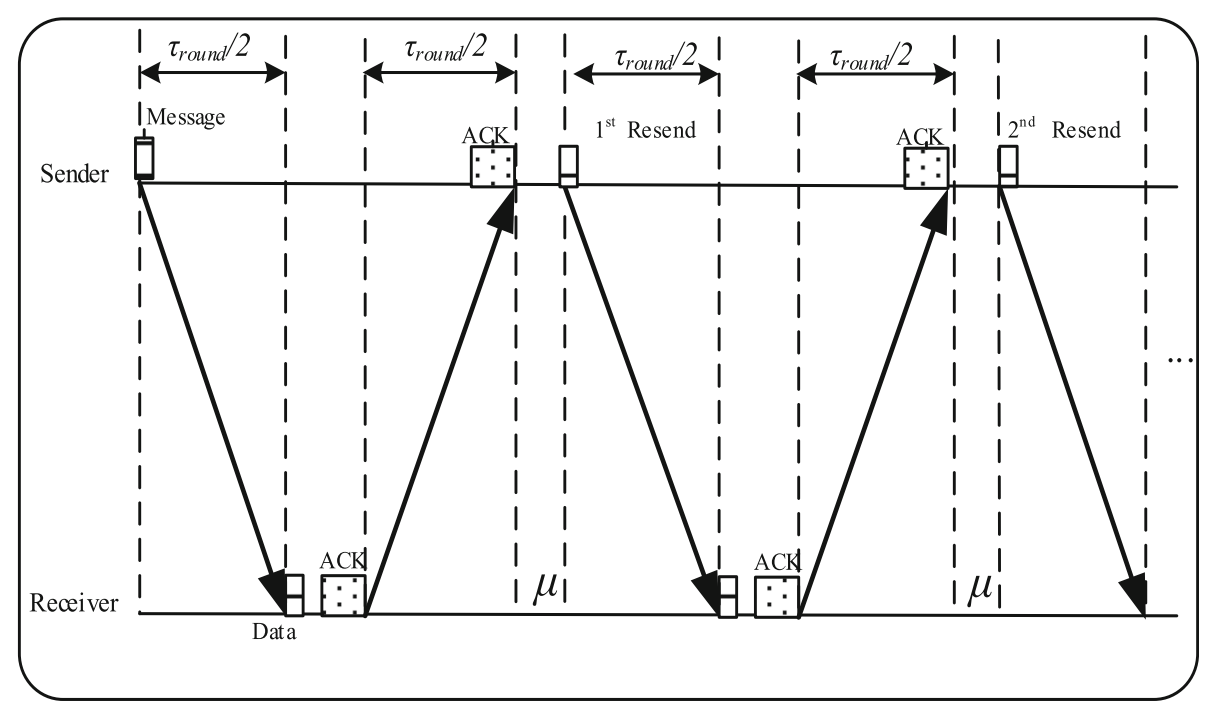

Fig. 2 SW-ARQ protocol timing diagram 
mechanism of this paper is a real differentiated service, capable of offering a variety of levels of service on a retransmission mechanism.

(3) The ARM-DDS scheme proposed in this paper has a different strategy than the previous one: We analyzed that the near-sink area in the wireless sensor network bears a large amount of data, so its energy consumption is large, which is hotspots. The energy of the hotspots area node has a residual, so in the ARM-DDS scheme, we fully increase the residual energy of these far-sink areas to appropriately increase the value of $c$ in the retransmission protocol and use a smaller $c$ value in the near-sink area. By making full use of the remaining energy of the network and improving energy utilization, it can increase the network lifetime under the same conditions as the previous strategy or further reduce the delay when the network life is the same.

Based on the above three points, the ARM-DDS protocol uses differentiated services for different requirements data to reduce transmission delay. When transmitting data streams with different reliability requirements, set different maximum retransmission times for different required data streams and the maximum number of retransmissions near the sink, improving network life, meeting higher reliability requirements, and improving energy utilization at far-sink nodes.

\subsection{General design of ARM-DDS}

In this section, the algorithm of the ARM-DDS scheme differentiated service is given by analyzing the data transmission mode.
Figures 2 and 3 represent the difference and improvement between the ARM-DDS strategy and the traditional SW-ARQ protocol. The data transmission of the SW-ARQ protocol is shown in Fig. 2. The sender sends a packet every time it transmits. Sending a packet to the receiver requires $\frac{1}{2} \tau_{\text {round }}$ time. The success rate is $p_{i}^{s}$. Receiver immediately sends an ACK to the sender when a packet is received. The ACK also needs $\frac{1}{2} \tau_{\text {round }}$ time to the sender. The rate is $q_{i}^{a}$. When sender receives the $\mathrm{ACK}$, it can be determined that the data transmission is successful. After sender sends the data packet, if the ACK is not received after waiting for $\tau_{\text {round }}+\mu$ time, it is considered that the sending of the data packet failed, and then sender needs to send the data packet again. Therefore, under the SW-ARQ protocol, additional $\tau_{\text {round }}+\mu$ time is required for each data retransmission. The sender will resend the data. Therefore, the SWARQ protocol requires $\tau_{\text {round }}+\mu$ time for each data to be retransmitted.

The ARM-DDS scheme proposed in this paper can continuously send multiple sets of data. When using ARM-DDS scheme transmission, first determine the $c$ value according to the requirements of the application. As shown in Fig. 3, the time when the data packet is sent to the receiver is $\frac{1}{2} \tau_{\text {round }}$, which is the same as the traditional protocol. When sending $c$ packets in succession, the interval of sending each packet is $s$. Therefore, when the packets of group $c$ arrive at receiver, the time is $\frac{1}{2}$ $\tau_{\text {round }}+(s-1) c$. The algorithm of receiver and the SWARQ protocol is consistent, and when a packet is received, an ACK is returned. The minimum interval for sending ACK is $b$. As long as one ACK is received by

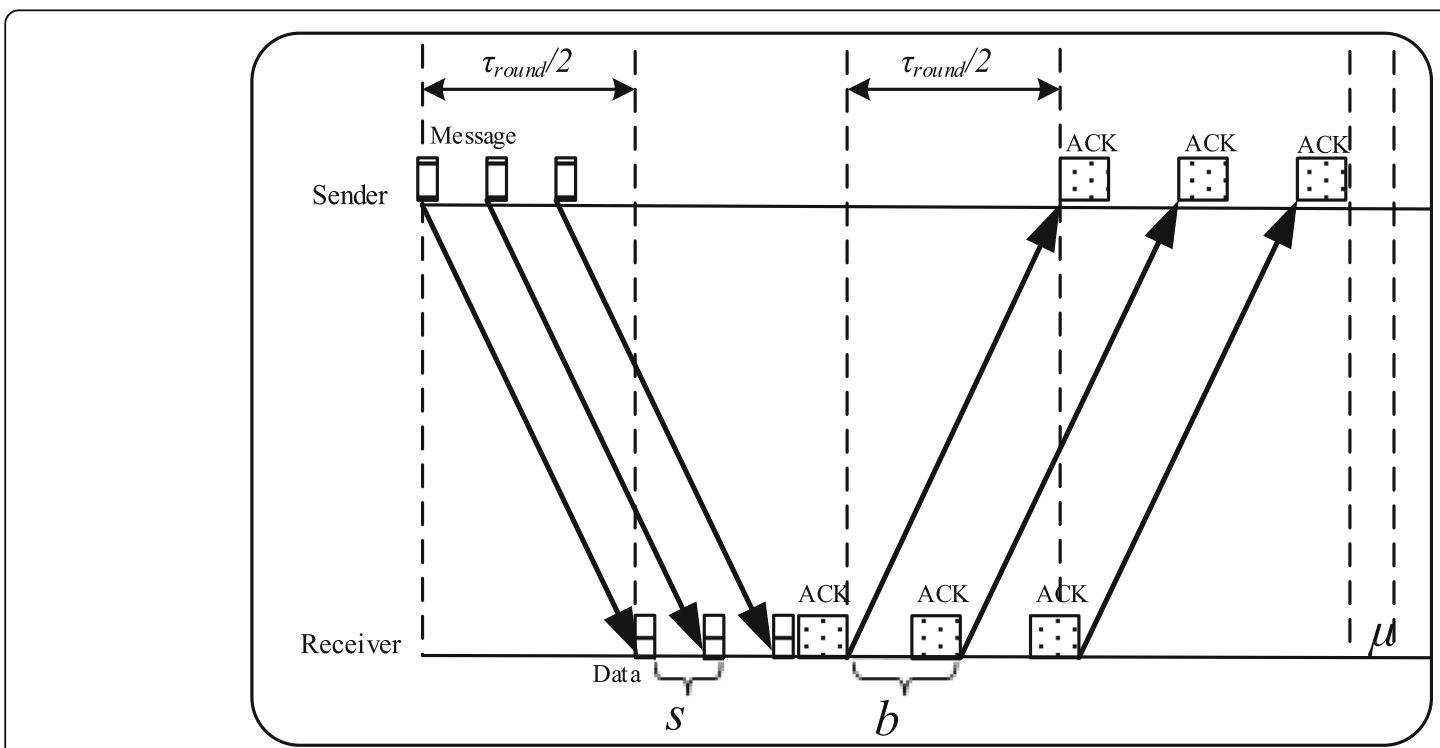

Fig. 3 ARM-DDS protocol timing diagram 
sender, it is regarded as successful. The transmission of the $c$-group data packets using the transmission strategy of the ARM-DDS scheme requires $\tau_{\text {round }}+(s+b-2) c$ delay. If the traditional protocol is used, this delay becomes $\left(\tau_{\text {round }}+\mu\right)$ c. In the network, the data transmission time $\tau_{\text {round }}$ is much larger than the minimum interval between data and ACK transmission $(s+b-2)$. Therefore, according to the above analysis, it can be obtained. The following conclusions are drawn: (1) When the number of retransmissions is small, the time required for the ARM-DDS protocol to transmit data with the SW-ARQ protocol is similar.

(2) When multiple retransmissions are required, the transmission delay of ARM-DDS will increase $(s+b-$ 2)c. The transmission delay of the SW-ARQ protocol is increased by $\left(\tau_{\text {round }}+\mu\right) c$. At this point, ARM-DDS has an advantage in latency. That is to say, the ARM-DDS scheme has a greater advantage in delay when it is required to retransmit data multiple times.

The algorithm of the ARM-DDS scheme is as follows: In a planar wireless sensor network, all nodes are awake and all nodes know their next hop node. When transmitting data, depending on the timeliness of the data, the data has different delay requirements. Time-sensitive data needs to arrive as quickly as possible, so the node must transmit the data as much as possible to ensure that its latency is low. Data with relatively low timeliness does not require more energy. Distinguish data with latency requirements so that each data can be transmitted within its required delay.

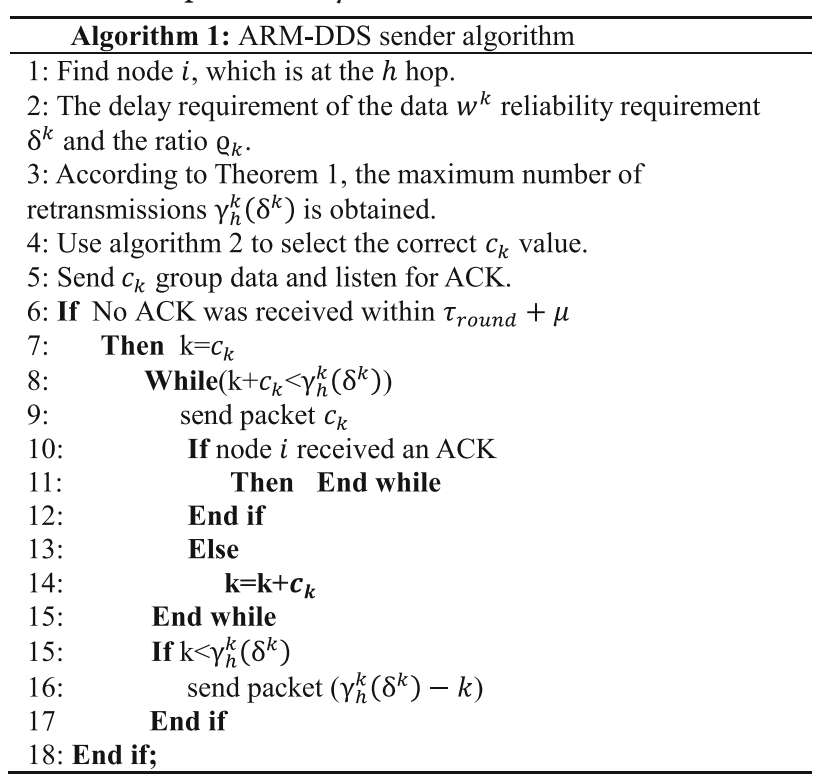

The algorithm of the receiving node is as follows: In the ARM-DDS protocol, the receiving node waits to receive a data packet, and if a group of data is received, it sends an ACK, otherwise, it continues to listen to the data packet.
The preceding discusses the transmission and reception methods of the ARM-DDS protocol. Now, the optimal value of the number of packets $c$ transmitted each time under the differentiated service is discussed. Therefore, the calculation of the optimal value has the following algorithm:

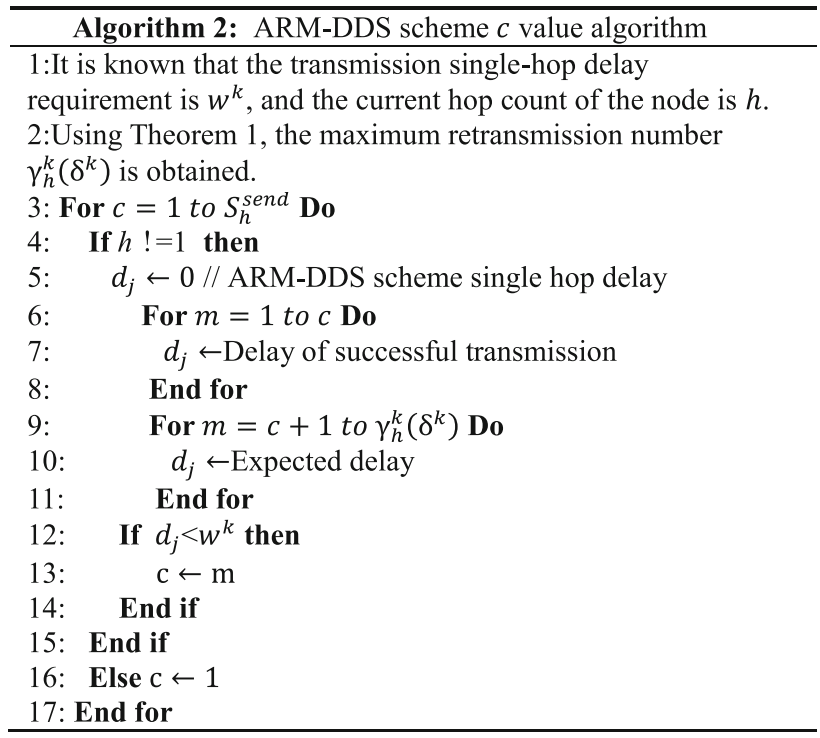

Algorithm 2 assigns the appropriate $c$ value to applications according to different delay requirements. The ARM-DDS protocol does not send multiple sets of data at one time to hotspots in the near-sink area. Because hotspots increase the number of sending groups, the energy consumption increases, the node death rate increases, and the network life decreases, which is undesirable for the research. Therefore, for a node with a hop count of 1 , it is consistent with the SW-ARQ protocol, that is, $c=1$. In this way, it is possible to differentiate the data stream without reducing the network life and reduce the purpose of delay.

Figure 4 is an E2E delay comparison of the two protocols. The experimental environment is a planar network with a radius of $400 \mathrm{~m}$ centered on the sink node. Its emission radius is $80 \mathrm{~m}$, reliability $\delta=0.8, p_{i}^{s}=0.8, q_{i}^{a}=0$ .8. As can be seen from Fig. 3, the ARM-DDS protocol delay is less than the SW-ARQ delay. And the further away from the sink, the greater the delay is reduced. When applications require different delays, the ARMDDS policy can change the value of $c$ to cause the data to be sent with the expected delay.

Regarding the loss of energy, the energy consumption of the ARM-DDS protocol proposed in this paper will be even larger. Because the ARM-DDS protocol increases the number of data transmissions compared to the SW-ARQ protocol, energy consumption is greater. Studies have shown that in a traditional flat network, when the lifetime of a network is exhausted, the energy 


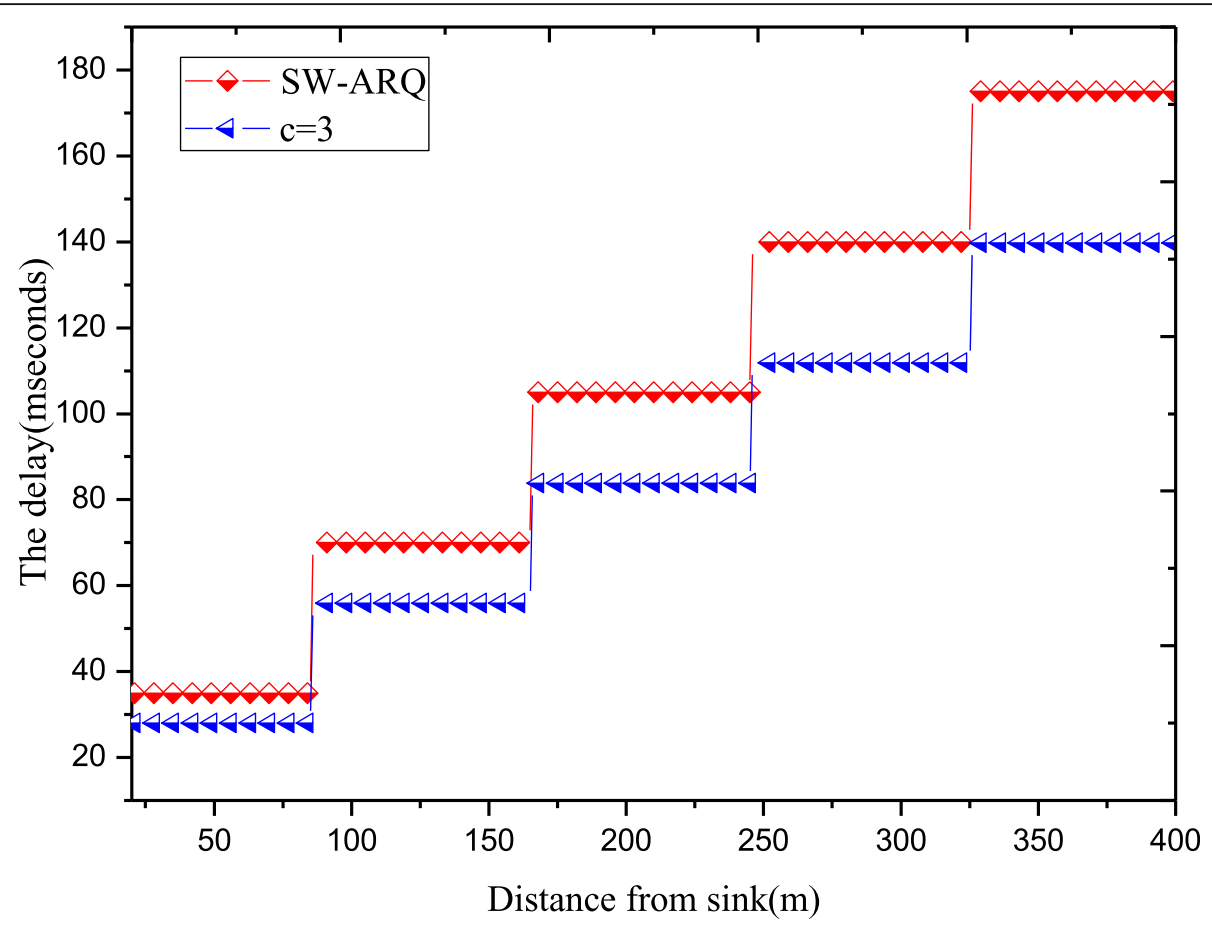

Fig. 4 End-to-end delay comparison between SW-ARQ and ARM-DDS scheme

remaining in the entire network is still as high as $90 \%$ [51]. Therefore, the ARM-DDS strategy can make full use of the remaining energy and increase the number of transmission groups at the far-sink without losing the network lifetime. Use this method to increase the energy utilization of the entire network [52, 53]. Therefore, adopting the above method does not affect the lifetime and can reduce the delay. Under the optimization of the differentiated service, the energy utilization of the entire network can also be increased.

\subsection{Parameter optimization of ARM-DDS protocol}

In the previous section, we mainly studied the transmission algorithm and $c$ value algorithm of ARM-DDS scheme. This section mainly discusses two other important parameters of the differentiated service: the data volume $\xi_{i}$ and the maximum number of retransmissions $\gamma_{h}^{\mathrm{ARM}}(\delta)$. Among them, $\gamma_{h}^{\mathrm{ARM}}(\delta)$ is the main parameter affecting network delay, and the data quantity $\xi_{i}$ is the main parameter affecting energy consumption.

When studying the difference in data volume between the ARM-DDS scheme and the traditional protocol, first analyze the data situation of the nodes in the network under the assumption that the data between the nodes is not lost, to provide a calculation for the case where the data loss rate between nodes is $>0$, the basics.

According to the reference [16], when the node transmits data with the transmission radius $r$, if the data transmission between the nodes is not lost, the distance of the node $i$ from the sink is $d, d=h r+\Delta$ (where $\Delta$ is a less than $r$ Constant), then the amount of data borne by the node $\xi_{i}$ is shown as equation (7).

$$
\begin{aligned}
& \xi_{i}=\frac{x(x+1)}{2 d} r+x+1 \\
& x=\left\lfloor\frac{R-\Delta}{r}\right\rfloor
\end{aligned}
$$

In a planar wireless sensor network, all data must have certain reliability requirements when it reaches the sink [54]. The statistical reliability required for transmission to the sink is represented by $\delta$. The maximum number of retransmissions of the ARM-DDS scheme $\gamma_{h}^{\mathrm{ARM}}(\delta)$ is different from the maximum number of retransmissions in the legacy protocol. In the SW-ARQ protocol, when applications with different reliability $\delta$ requirements are simultaneously transmitted since the service cannot be distinguished, all data can be transmitted only according to the maximum $\delta$. This will cause the data that has already met $\delta$ to be retransmitted, the excess energy is wasted, and the delay is added. ARM-DDS scheme was proposed to optimize the above situation. The protocol can reasonably distinguish between applications with different $\delta$ requirements and set the appropriate number of retransmissions for each application.

For any node $i$ in the planar network, the success rate of the node per-hop transmission is $p_{i}^{s}$. According to the 
reference, in the planar wireless sensor network, the SW-hop-by-hop ARQ protocol is used, and the node with the hop count $h$ is used. When the maximum number of retransmissions is as follows, the reliability is guaranteed to be $\delta$ :

$$
\gamma_{h}(\delta)=\left\lceil\frac{\log \left(1-\delta^{\frac{1}{h+1}}\right)}{\log \left(1-p_{i}^{s}\right)}\right\rceil
$$

The biggest difference between the ARM-DDS protocol and the SW-ARQ protocol is the maximum number of retransmissions. Under the differentiated service, the maximum number of retransmissions of the ARM-DDS protocol must be less than or equal to the SW-ARQ protocol. Theorem 1 gives the maximum number of retransmissions $\gamma_{h}^{\mathrm{ARM}}(\delta)$ of the ARM-DDS protocol.

Theorem 1: In the ARM-DDS protocol, it is assumed that there are $m$ kinds of data packets with different reliability requirements $\delta^{k}$ in the data stream to be transmitted, each ratio $\mathrm{Q}_{k} \%$ (where $k$ is the number of different data streams, $0 \leq k \leq m$ ), the maximum number of retransmissions under different data reliability requirements is $\gamma_{h}^{k}\left(\delta^{k}\right)$, so the maximum number of retransmissions under the

$$
\gamma_{h}^{\mathrm{ARM}}(\delta)=\left\lceil\sum_{k=1}^{m} \gamma_{h}^{k}\left(\delta^{k}\right) \mathrm{e}_{k}\right\rceil
$$

Proof In the SW-hop-by-hop ARQ transmission mode, the maximum number of retransmissions of a hop is $\gamma_{h}(\delta)$. Based on this, the ARM-DDS protocol differentiates the data streams with different reliability requirements. When the reliability requirement is $\delta^{k}, \gamma_{h}^{k}\left(\delta^{k}\right)$ times need to be retransmitted, and the data stream is the proportion of the overall data stream which is $\varrho_{k} \%$, so we can conclude that the maximum retransmission number of the data stream with the reliability requirement of $\delta^{k}$ is $\gamma_{h}^{k}\left(\delta^{k}\right) \varrho_{k}$. Similarly, all the different reliability requirements data will be obtained. The flow is weighted and calculated:

$$
\gamma_{h}^{\mathrm{ARM}}(\delta)=\left\lceil\sum_{k=1}^{m} \gamma_{h}^{k}\left(\delta^{k}\right) \varrho_{k}\right\rceil
$$

In the previous, the retransmission times and data amount parameters of the ARM-DDS protocol are proposed. To get the influence of these parameters on the whole network, it is necessary to calculate the amount of data and the amount of ACK when transmitting data and analyze the difference between the ARM-DDS scheme and the SW-ARQ protocol data transmission.

In the SW-ARQ protocol, the number of data packets and the amount of information that each node bears are as follows:
Let the distance of the node $i$ from the sink be $d$; then, we know that $d=r \times h+\Delta$ if the transmission and reception (SW-ARQ) mode are used. Considering that the reliability required for transmission is $\delta$, the data commitment amount $D_{\xi, i}^{h, t}$ of the node $i$, and the ACK amount $X_{\xi, i}^{h, t}$ are

$$
\begin{aligned}
& D_{\xi, i}^{h, t}=\mathrm{Z}_{h}^{h}(\delta)+\mathrm{Z}_{h+1}^{h}(\delta)\left(1+\frac{r}{d}\right)+\mathrm{Z}_{h+2}^{h}(\delta) \\
& \times\left(1+\frac{2 r}{d}\right) \ldots+\mathrm{Z}_{x}^{h}(\delta)\left(1+\frac{(x-h) r}{d}\right) \\
& X_{\xi, i}^{h, t}=D_{\xi, i}^{h-1, t} p_{i}^{s}=W_{h}^{h}(\delta)+W_{h+1}^{h}(\delta)\left(1+\frac{r}{d}\right) \\
& +W_{h+2}^{h}(\delta)\left(1+\frac{2 r}{d}\right) \\
& +\ldots W_{x}^{h}(\delta)\left(1+\frac{(x-h) r}{d}\right) \\
& D_{\xi, i}^{h, r}=D_{\xi, i}^{h-1, t}-\mathrm{Z}_{h}^{h}(\delta) \\
& X_{\xi, i}^{h, r}=X_{\xi, i}^{h, r}=D_{\xi, i}^{h, t} p_{i}^{s} q_{i}^{a} \\
& \mathrm{Z}_{h+j}^{h}(\delta)=\frac{1-\left(1-p_{i}^{s} q_{i}^{a}\right)^{\gamma_{h+j}(\delta)}}{p_{i}^{s} q_{i}^{a}} \\
& W_{h}^{h}(\delta)=0 \\
& W_{h+j}^{h}(\delta)=\mathrm{Z}_{h+j}^{h+1}(\delta) p_{i}^{s}
\end{aligned}
$$

Theorem 2: When multiple sets of data of ARM-DDS protocol are transmitted simultaneously, the amount of data is given as follows: According to Theorem 1, it is assumed that the hop count of node $i$ ish, considering the reliability requirement is $\delta^{k}$, and the maximum number of retransmissions is $\gamma_{h}^{k}\left(\delta^{k}\right)$. Therefore, the expected number of transmissions per hop $S_{h}^{\text {send }}$, the amount of reception $S_{h}^{\text {rec }}$, and the amount of ACK $S_{h}^{A R}$ and the number of transmission $S_{h}^{A S}$ are as follows:

$$
\begin{aligned}
S_{h}^{\text {send }}= & E_{h+0}^{h}\left(\delta^{k}\right) p_{i}^{s 0}+\left(1+\frac{r}{d}\right) E_{h+1}^{h}\left(\delta^{k}\right) p_{i}^{s 1} \\
& +\left(1+\frac{2 r}{d}\right) E_{h+2}^{h}\left(\delta^{k}\right) p_{i}^{s 2} \\
& +\ldots\left(1+\frac{(x-h) r}{d}\right) E_{x}^{h}\left(\delta^{k}\right) p_{i}^{s x-h} \\
S_{h}^{A S}= & S_{h}^{\mathrm{rec}}=p_{i}^{s}\left(\left(1+\frac{r}{d}\right) \cdot E_{h+1}^{h}\left(\delta^{k}\right) p_{i}^{s 1}+\right. \\
(1+ & \left.\frac{2 r}{d}\right) E_{h+2}^{h}\left(\delta^{k}\right) p_{i}^{s 2} \\
& +\ldots\left(1+\frac{(x-h) r}{d}\right) E_{x}^{h}\left(\delta^{k}\right) p_{i}^{s x-h}
\end{aligned}
$$




$$
S_{h}^{A R}=S_{h}^{\text {send }} p_{i}^{s} q_{i}^{a}
$$

Among them,

$$
\begin{aligned}
E_{j}^{h}\left(\delta^{k}\right)= & c_{h}+\sum_{v=1}^{\left\lfloor\gamma_{h}^{k}\left(\delta^{k}\right) / c_{h}\right\rfloor} v p_{i}^{s} q_{i}^{a}\left(1-\left(1-p_{i}^{s} q_{i}^{a}\right)^{c_{h}}\right)\left(1-p_{i}^{s} q_{i}^{a}\right)^{(v-1) c_{h}} \\
& +\gamma_{j}^{k}\left(\delta^{k}\right)\left(1-p_{i}^{s} q_{i}^{a}\right)^{\left\lfloor\gamma_{h}^{k}\left(\delta^{k}\right) / c_{h}\right\rfloor}
\end{aligned}
$$

Proof Because the ARM-DDS strategy is used, the node's data needs to be sent at least $c_{h}$ times. Therefore, the probability that the transmitted data has at least $c_{h}$ times is 1 . If at least one of the transmitted data is successfully transmitted, the data transmission will stop. Only when this $c_{h}$ data transmission fails will $c_{h}$ packets be sent again. The probability of successful transmission within $2 c_{h}$ times is $\left(1-\left(1-p_{i}^{s} q_{i}^{a}\right)^{c_{h}}\right)\left(1-p_{i}^{s} q_{i}^{a}\right)^{c_{h}}$.This means the first set of data transfers failed, but the second set of $c_{h}$ data transfers was successful. And sender also successfully received the ACK from the next hop. The probability that the second group $c_{h}$ transmission is successful is the probability that all the first $c_{h}$ group data transmission failures $\left(1-p_{i}^{s} q_{i}^{a}\right)^{c_{h}}$ are multiplied by the probability that the second group data is successfully sent to receive. And the probability that sender successfully receives the ACK is $1-\left(1-p_{i}^{s} q_{i}^{a}\right)^{c_{h}}$. By analogy, the probability of successful transmission in the first $v c_{h}$ is ( $\left.1-\left(1-p_{i}^{s} q_{i}^{a}\right)^{c_{h}}\right)\left(1-p_{i}^{s} q_{i}^{a}\right)^{(v-1) c_{h}}$. Until $(v+1) c_{h}$ is greater than $\gamma_{h}^{k}\left(\delta^{k}\right)$, the $\gamma_{h}^{k}\left(\delta^{k}\right)-\left\lfloor\gamma_{h}^{k}\left(\delta^{k}\right) / c_{h}\right\rfloor$ group data is continuously transmitted. Therefore, the expected value sent by the node is

$$
\begin{aligned}
E_{j}^{h}\left(\delta^{k}\right)= & c_{h}+\sum_{v=1}^{\left\lfloor\gamma_{h}^{k}\left(\delta^{k}\right) / c_{h}\right\rfloor} v p_{i}^{s} q_{i}^{a}\left(1-\left(1-p_{i}^{s} q_{i}^{a}\right)^{c_{h}}\right)\left(1-p_{i}^{s} q_{i}^{a}\right)^{(v-1) c_{h}} \\
& +\gamma_{j}^{k}\left(\delta^{k}\right)\left(1-p_{i}^{s} q_{i}^{a}\right)^{\left\lfloor\gamma_{h}^{k}\left(\delta^{k}\right) / c_{h}\right\rfloor}
\end{aligned}
$$

When a packet from an $h+j$ hop node is sent to a node with a hop count of $h$, the amount of data that the node needs to bear is: $\frac{(d+j r)}{d}$. Therefore, the number of packets sent from the node of $h+j$ hop to $h$ hop is $E_{h+j}^{h}\left(\delta^{k}\right) \frac{(d+j r)}{d}$. The probability of sending to $h$ hop is $p_{i}^{s j}$. Therefore, the number of packets sent by the node with the hop count is

$$
\begin{aligned}
S_{h}^{\text {send }}= & E_{h+0}^{h}\left(\delta^{k}\right) p_{i}^{s 0}+\left(1+\frac{r}{d}\right) E_{h+1}^{h}\left(\delta^{k}\right) p_{i}^{s 1} \\
& +\left(1+\frac{2 r}{d}\right) E_{h+2}^{h}\left(\delta^{k}\right) p_{i}^{s 2} \\
& +\ldots\left(1+\frac{(x-h) r}{d}\right) E_{x}^{h}\left(\delta^{k}\right) p_{i}^{s x-h}
\end{aligned}
$$

The number of ACKs that need to be sent is as follows: Each node receives an ACK and sends an ACK. Therefore, the number of ACKs sent is equal to the number of received packets. The number of received packets is the number of data sent by the previous node multiplied by $p_{i}^{s}$ (because there is a packet loss). Therefore, there is the following formula:

$$
\begin{aligned}
S_{h}^{A S}=S_{h}^{\mathrm{rec}} & =p_{i}^{s}\left(\left(1+\frac{r}{d}\right) E_{h+1}^{h}\left(\delta^{k}\right) p_{i}^{s}+\left(1+\frac{2 r}{d}\right) E_{h+2}^{h}\left(\delta^{k}\right) p_{i}^{s 2}\right. \\
& \left.+\ldots\left(1+\frac{(x-h) r}{d}\right) E_{x}^{h}\left(\delta^{k}\right) p_{i}^{s x-h}\right)
\end{aligned}
$$

Considering that the number of packets sent by the node $i$ with the hop count $h$ to the next hop is $S_{h}^{\text {send }}$, the packets arriving at the next hop are $p_{i}^{s}$. Each packet is received by the receive and returns an ACK. The probability that the return ACK is successfully received by sender is $q_{i}^{a}$. Therefore, the number of ACKs received by the node is

$$
S_{h}^{A R}=S_{h}^{\text {send }} p_{i}^{s} q_{i}^{a}
$$

As can be seen from Section 5.2, the larger the $c$ value, the smaller the transmission delay. In the ARM-DDS scheme, if the $c$ value is greater than 1 , the transmission delay compared to the SW-ARQ protocol will be reduced. In the ARM-DDS protocol, since the nonhotspots area has more energy remaining, we can set the non-hotspots area to have a larger $c$ value. This reduces transmission delays, ensures network lifetime, and increases energy utilization.

In Section 5.3, it can be concluded from Theorem 1, that the number of retransmissions of the ARM-DDS scheme is less than or equal to the traditional SW-ARQ protocol. In the performance of the data amount, according to Algorithm 2 and Theorem 2, it can be concluded that since the ARM-DDS strategy uses a larger $c$ value in the non-hotspots area, the amount of transmitted and received data is higher than the SW-ARQ protocol. In the hotspots area, the number of retransmissions of the ARM-DDS policy is smaller than that of the SWARQ protocol, and the number of data transmitted each time is equal to the conventional protocol. Therefore, the data amount of the ARM-DDS policy at this time is smaller than the SW-ARQ protocol.

\section{The experimental results and analysis}

This section mainly analyzes the two most important factors of the ARM-DDS protocol for the network: the impact of transmission delay and energy consumption. Discuss the performance of the ARM-DDS protocol in these two aspects under differentiated services.

\subsection{Transmission delay}

Theorem 3: In the ARM-DDS protocol, the node $i$ of any $h$ hop and its distance to the sink is $d, d=h r+\Delta$. 
The end-to-end delay $\omega_{h}$ is as follows when transmitting data with different delay requirements:

$$
\begin{aligned}
\omega_{h}= & \sum_{j=1}^{h} w_{h 2 j} \\
w_{h 2 j}= & \sum_{v=1}^{c_{j}}\left(p_{i}^{s} \prod_{m=1}^{v}\left(1-p_{i}^{s}\right)\left(s(m-1)+\frac{1}{2} \tau_{\text {round }}\right)\right) \\
& +\sum_{v=c_{j}+1}^{2 c_{j}}\left(p_{i}^{s} \prod_{m=1}^{v}\left(1-p_{i}^{s}\right)\left(s(m-1)+\frac{1}{2} \tau_{\text {round }}\right)\right) \\
& +\ldots \sum_{v=(k-1) c_{j}+1}^{\left\lfloor\gamma_{h}^{k}\left(\delta^{k}\right) / c_{j}\right.}\left(p_{i}^{s} \prod_{m=1}^{v}\left(1-p_{i}^{s}\right)\left(s(m-1)+\frac{1}{2} \tau_{\text {round }}\right)\right) \\
& +\sum_{v=\left\lfloor\gamma_{h}^{k}\left(\delta^{k}\right) / c_{j}\right\rfloor+1}^{\gamma_{j}(\delta)}\left(p _ { i } ^ { s } \prod _ { m = 1 } ^ { v } ( 1 - p _ { i } ^ { s } ) \left(\frac{1}{2} \tau_{\text {round }}+\mu\right.\right. \\
& \left.\left.+\left(c_{j}-1\right)(s+b)+\tau_{\text {rtto }}\left(v-c_{j}-1\right)\right)\right)
\end{aligned}
$$

Proof According to Theorem 1, under the delay required by the application, the maximum number of retransmissions of the node of the $h$ hop is $\gamma_{h}^{k}\left(\delta^{k}\right)$. The transmission delay can be expressed as $w_{h}=\sum_{v=0}^{\gamma_{h}^{k}\left(\delta^{k}\right)} p_{v}$ $P_{v}$, where $p_{v}$ is the probability of successfully transmitting a packet for the $v$ time, and $P_{v}$ is the delay of the $v$ th successful transmission. When the node sends data to the next hop, all packets need to arrive: $s\left(c_{h}-1\right)+\frac{1}{2}$ $\tau_{\text {round }}$. Where $c_{h}$ is the number of consecutively transmitted packets for the $h$ hop, and $s$ is the data sent each time interval between. If the first set of $c_{h}$ data transmission fails, the sender will transmit the second set of data of $c_{h}$ until the maximum number of retransmissions is less than $c_{h}$ times.

Therefore, we can conclude that the probability of successful arrival at the $v$ th $\left(v \leq \gamma_{h}(\delta)\right)$ transmission is $p_{i}^{s}$ $\prod_{m=1}^{v}\left(1-p_{i}^{s}\right)$. The maximum expected delay after receive processing the data packet is $\frac{1}{2} \tau_{\text {round }}+\mu+\left(c_{h}-1\right)(s+b)$ $+\tau_{\text {rtto }}\left(v-c_{h}-1\right)$.

In summary, the delay for $h$ hop to jump to $j$ is as follows:

$$
\begin{aligned}
w_{h 2 j}= & \sum_{v=1}^{c_{h}}\left(p_{i}^{s} \prod_{m=1}^{v}\left(1-p_{i}^{s}\right)\left(s(m-1)+\frac{1}{2} \tau_{\text {round }}\right)\right) \\
& +\sum_{v=c_{j}+1}^{2 c_{h}}\left(p_{i}^{s} \prod_{m=1}^{v}\left(1-p_{i}^{s}\right)\left(s(m-1)+\frac{1}{2} \tau_{\text {round }}\right)\right) \\
& +\ldots \sum_{v=(k-1) c_{i}+1}^{k c_{h}}\left(p_{i}^{s} \prod_{m=1}^{v}\left(1-p_{i}^{s}\right)\left(s(m-1)+\frac{1}{2} \tau_{\text {round }}\right)\right) \\
& +\sum_{v=k c_{j}+1}^{\gamma(\delta)}\left(p _ { i } ^ { s } \prod _ { m = 1 } ^ { v } ( 1 - p _ { i } ^ { s } ) \left(\frac{1}{2} \tau_{\text {round }}+\mu\right.\right. \\
& \left.\left.+\left(c_{h}-1\right)(s+b)+\tau_{\text {rtto }}\left(v-c_{h}-1\right)\right)\right)
\end{aligned}
$$

The end-to-end delay of the $h$-th hop is

$$
\omega_{h}=\sum_{j=1}^{h} w_{h 2 j}
$$

Theorem 4: In a planar wireless sensor network with radius $R$, the known transmission radius is $r$ and the node distribution density is $\rho$. The average end-to-end delay per hop is $\omega_{h}$. The average weighted delay for the entire network is as follows:

$$
\omega=\sum_{h=1}^{h=x} \frac{\left[(h r)^{2}-((h-1) r)^{2}\right] \omega_{h}}{R^{2}}
$$

Proof Suppose the radius of the planar network is $R$, the radius per hop is $r$, the density of nodes is $\rho$, and the end-to-end delay per hop is $\omega_{h}$. Since each hop of the planar network can be approximated as a ring shape, the area of the $h$ th hop can be found as $\pi(h r)^{2}-\pi((h-1) r)^{2}$. Therefore, the node of the $h$ th jump has a total of $\rho\left[\pi(h r)^{2}-\pi((h-1) r)^{2}\right]$, and the average weighted delay of the entire planar network is

$$
\sum_{h=1}^{h=x} \frac{\rho\left[(h r)^{2}-((h-1) r)^{2}\right] \omega_{h}}{\pi R^{2} \rho}
$$

which is

$$
\sum_{h=1}^{h=x} \frac{\left[(h r)^{2}-((h-1) r)^{2}\right] \omega_{h}}{R^{2}}
$$

This paper uses the above formula theorem to calculate the performance of the ARM-DDS protocol in terms of network transmission delay.

\subsection{Energy consumption}

In WSNs, nodes in the non-hotspots area still have a lot of energy left when the network life expires, causing waste. Therefore, the remaining energy of the nonhotspots region node can be fully utilized to set a larger $c$ value and reduce the transmission delay. The nodes of hotspots need not lose excess energy. Therefore, setting $c=1$ does not reduce the network lifetime. The following is the theorem about network energy consumption.

Theorem 5: In the ARM-DDS protocol, considering that the energy consumption of nodes transmitting and receiving data packets is $e_{p}^{t}$ and $e_{p}^{r}$, respectively, the energy consumption of transmitting and receiving ACKs by nodes is $e_{A}^{t}$ and $e_{A}^{t}$, respectively. Then the energy consumption of the $h$-th hop node is as follows:

$$
E_{h}^{i}=\mathrm{S}^{\mathrm{send}} e_{p}^{t}+\mathrm{S}^{\mathrm{rec}} e_{p}^{r}+\mathrm{S}^{A S} e_{A}^{t}+\mathrm{S}^{A R} e_{A}^{r}
$$

Proof From Theorem 2, the transmission received data packet $S_{h}^{\text {send }}$ and the reception amount $S_{h}^{\text {rec }}$ and the ACK bearer $S_{h}^{A R}$ and the transmission amount $S_{h}^{A S}$ are obtained.

So the energy consumption of a node is the product of the number of transmissions and the energy required to transmit each packet: 


$$
o_{1}^{i}=\mathrm{S}^{\mathrm{send}} e_{p}^{t}+\mathrm{S}^{\mathrm{rec}} e_{p}^{r}+\mathrm{S}^{A S} e_{A}^{t}+\mathrm{S}^{A R} e_{A}^{r}
$$

Theorem 5 is given to provide a theoretical basis for network lifetime. Investigating the energy consumption of hotspot nodes helps determine network lifetime.

This article does not consider low probability burst situations. Assume that all nodes consume the same amount of energy per round. After deriving the energy consumption of all nodes in the network, the network lifetime can be given.

Theorem 6: Assuming that the lifetime of a planar network is $\mathcal{L}$, the total energy of the nodes in the hotspots region $\mathcal{O}_{1}^{\text {all }}$ is related to the energy consumption $o_{1}^{i}$

. We can get the following formula:

$$
\mathcal{L}=\frac{\mathcal{O}_{1}^{\text {all }}}{o_{1}^{i}}=\frac{\mathcal{O}_{1}^{\text {all }}}{S_{1}^{\text {send }} e_{p}^{t}+S_{1}^{\text {rec }} e_{p}^{r}+S_{1}^{A S} e_{A}^{t}+S_{1}^{A R} e_{A}^{r}}
$$

The subscript refers to the hotspot area node closest to the sink. The average lifetime of these nodes determines the lifetime of the entire network.

Proof Because in a flat network, we can know from the energy consumption formula that the energy consumption of the near-sink node is the largest. Once the network has energy-saving exhaustion, it means that the planar network is exhausted. Assume that in one cycle, the energy consumption of the near-sink node is $o_{1}^{i}$ $=\mathrm{S}_{1}^{\mathrm{send}} e_{p}^{t}+\mathrm{S}_{1}^{\mathrm{rec}} e_{p}^{r}+\mathrm{S}_{1}^{A S} e_{A}^{t}+\mathrm{S}_{1}^{A R} e_{A}^{r}$, the total energy of the node is $\mathcal{O}_{1}^{\text {all }}$. Therefore, in the case of constant physical conditions, the node near the sink can run the $\mathcal{L}$ $=\frac{\mathcal{O}_{1}^{\text {all }}}{o_{1}^{i}}$ period, after which the node will run out of energy, so define it as the lifetime of the entire network.

This paper uses the above formula theorem to calculate the performance of the ARM-DDS protocol in terms of network energy consumption.

\section{Simulation and analysis results}

In a planar wireless sensor network, the one-hop delay of a packet refers to the time it takes from being sent by the current node to being received by the next hop node. The end-to-end delay of a packet is the time it takes to be transmitted from the source node to the sink. In this section, the experimental results of the ARM-DDS protocol and the SW-ARQ protocol are given.

The experimental parameters of this paper are set as follows: $R=500, r=80, p_{i}^{s}=0.8, q_{i}^{a}=0.8, \quad \tau_{\text {round }}=5$ $0 \mathrm{~ms}$. The number of data bits is $100 \mathrm{~b}$, and the number of ACK bits is $10 \mathrm{~b}$. The latency and reliability requirements of the data stream are as follows:

When $\delta=0.8$, the one-hop delay requirement is $25 \mathrm{~ms}$ and accounts for $30 \%$ of the data stream.
When $\delta=0.9$, the one-hop delay requirement is $30 \mathrm{~ms}$ and accounts for $50 \%$ of the data stream;

When $\delta=0.95$, the one-hop delay requirement is $35 \mathrm{~ms}$ and accounts for $20 \%$ of the data stream.

\subsection{Delay comparison}

The comparison between the traditional SW-ARQ protocol and the ARM-DDS protocol in terms of delay is given in this section. When analyzing the transmission performance of the two protocols, the $c$ value of the ARM-DDS protocol should be determined first.

Algorithm 2 gives the value of $c$ in different network environments, as shown in Fig. 4. The end-to-end delay at different $c$ values is given in Fig. 5. Combined with Figs. 4 and 5, the following conclusions are given: (1) The lower the data transmission delay, the larger the value of $c$. The reason is that the larger the number of simultaneous transmission groups $c$, the smaller the expected time required for successful transmission. Therefore, when transmitting time-sensitive data, the value of $c$ will be larger. (2) The reduced delay of the ARM-DDS protocol is limited. This is because if the $c$-group data is sent at the same time, and the transmission time is increased by $(c-1) s$. When the value of $c$ is too large, an increase in the transmission time causes an increase in delay.

Therefore, setting an appropriate $c$ value is especially important.

Figures 6 and 7 show the one-hop delay of the ARMDDS protocol and the SW-ARQ protocol under different data streams with different delay requirements. From Fig. 6, the following conclusions can be drawn:

(1) In the SW-ARQ protocol, considering that the reliability of the data reaching the sink is $\delta$, the number of retransmissions of the far-sink nodes is greater than the number of retransmissions of the near-sink nodes. The reason is that the data sent by the node of the far-sink area has to go through more hops before it reaches the sink. To ensure data reliability, it must be retransmitted more times. Therefore, the delay of far-sink nodes is higher than the near-sink node.

(2) In terms of one-hop delay of the near-sink node, both protocols use "one transmission and one reception" data transmission method, so the one-hop delay of the two is the same.

(3) When the number of transmission hops is greater than 1, the ARM-DDS protocol will send multiple sets of data each time, reducing the amount of time it takes to listen to the ACK (as shown in Fig. 3). Therefore the ARM-DDS protocol has a lower single-hop delay. When the SW-ARQ protocol can meet the delay requirement, the maximum number 


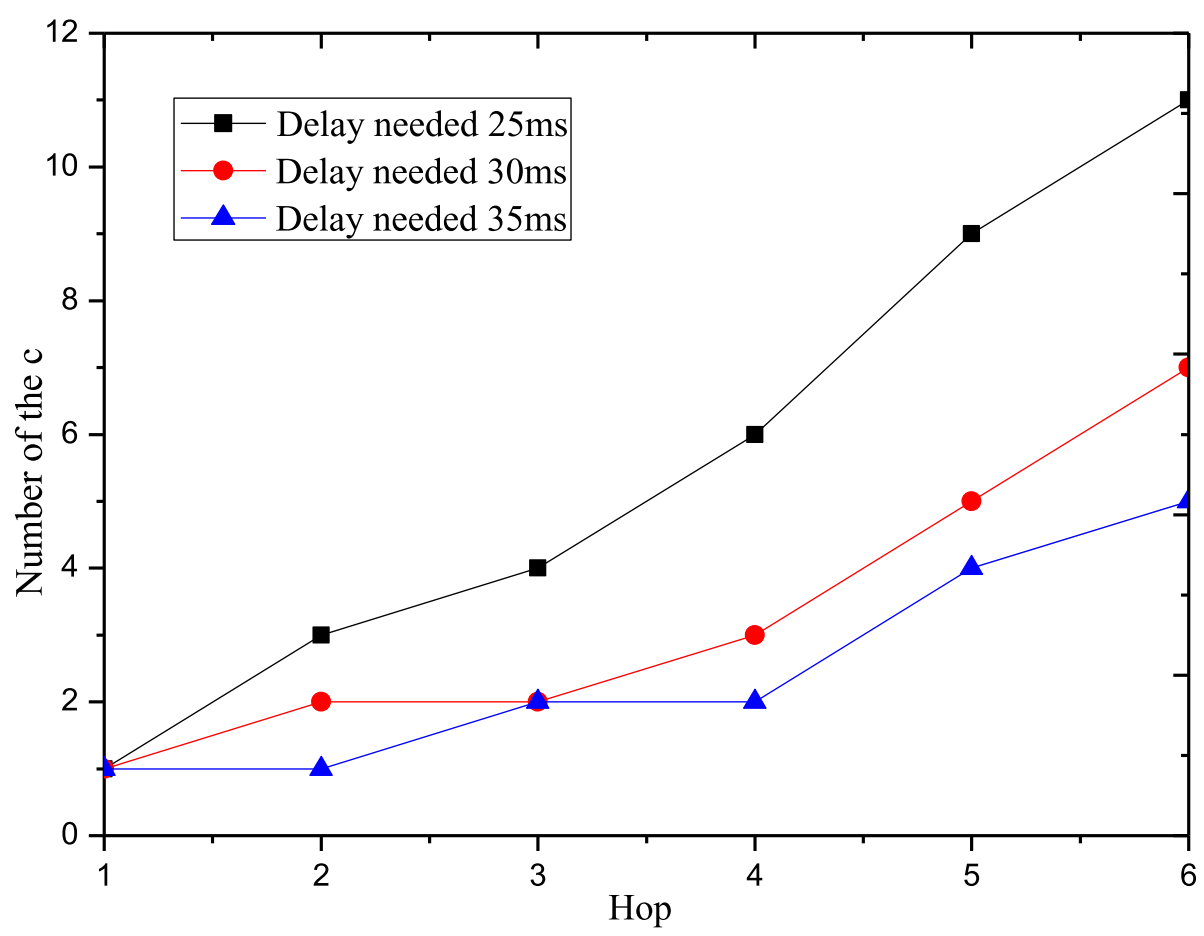

Fig. 5 Optimal $c$ value per hop $\left(p_{i}^{s}=0.8\right)$

of retransmissions of the data can only be set according to the highest reliability requirement in the data stream.

(4) The delay of the DMDT protocol fluctuates because of the network environment setting and the integer value of $c$. Finally, as the value of $c$ becomes larger, the one-hop delay will be significantly reduced.

(5) Therefore, the SW-ARQ protocol delay will still be greater than the ARM-DDS protocol. Therefore, the one-hop delay of the ARM-DDS protocol is less

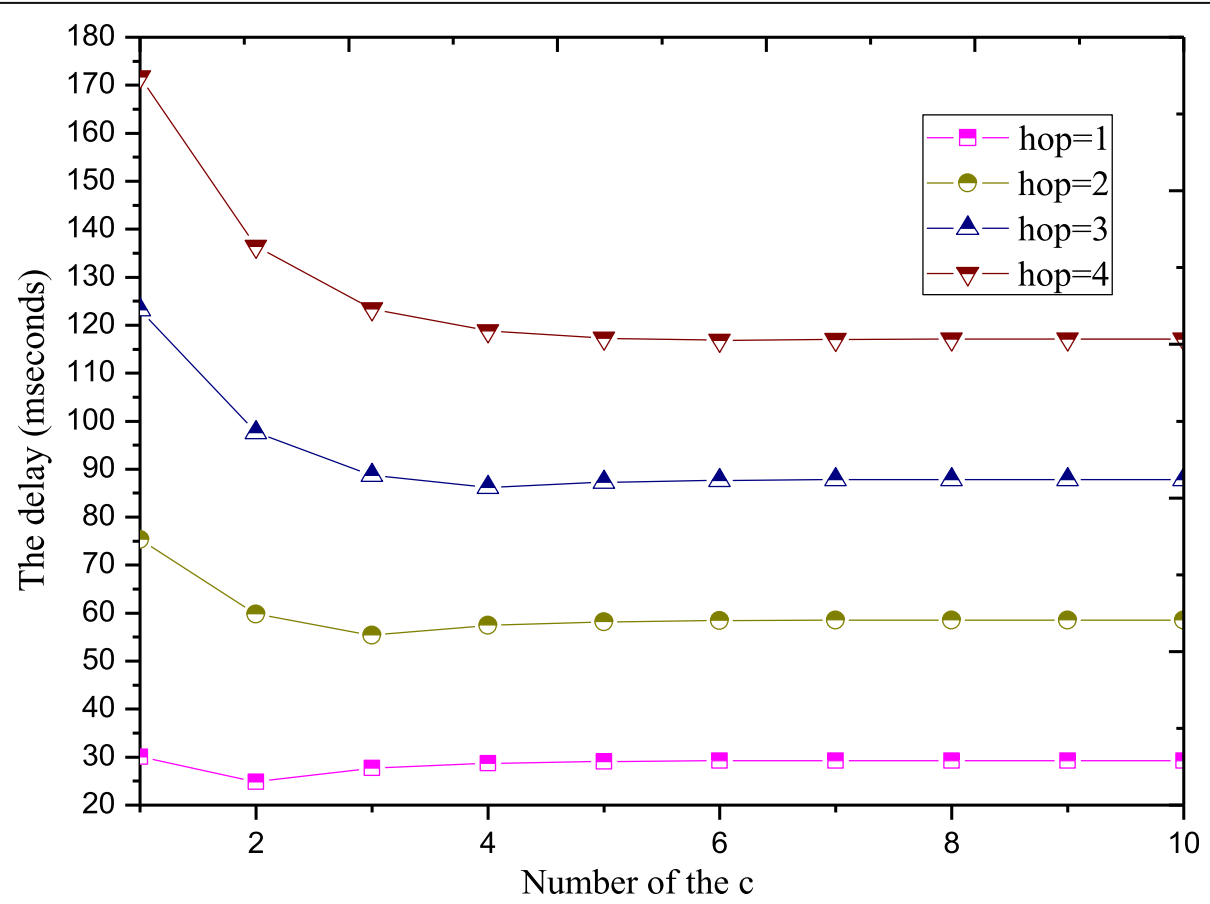

Fig. 6 Delay variation per hop $\left(p_{i}^{s}=0.8\right)$ 


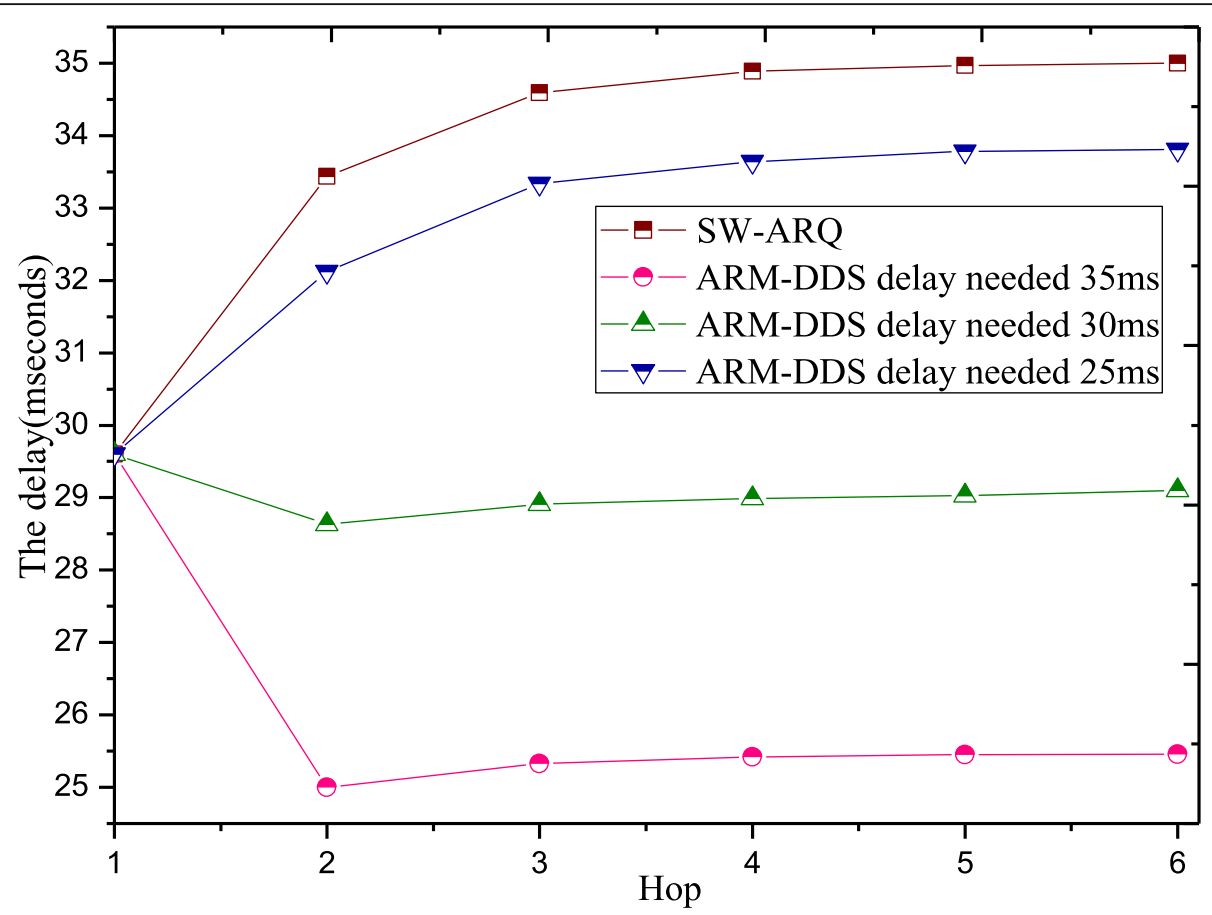

Fig. 7 Comparison of single-hop delays for different protocols

than or equal to the SW-ARQ protocol, which indicates that the ARM-DDS protocol can effectively reduce the transmission delay compared to the SWARQ protocol.

Figures 8 and 9 are end-to-end (E2E) delays at different success rates $p$. The following can be seen from the figures:

(1) Nodes near the sink are insensitive to the change of the transmission success rate $p$, because nodes near the sink only need to transmit data once. Therefore, when the transmission success rate $p$ changes, the influence on the first hop is minimal.

(2) It can be seen from Fig. 8 that as the value of $p$ becomes smaller, the maximum number of retransmissions per hop becomes larger. Because of the lower transmission success rate, the probability of data transmission failure increases, and the probability of retransmission increases. As shown in the figures, the end-to-end delays of both protocols increase as $p$ becomes smaller.

(3) As the value of $p$ becomes smaller, the ARM-DDS protocol reduces delay greater than the SW-ARQ protocol.

Therefore, the worse the network environment the ARM-DDS protocol works in, the more the transmission delay is reduced.
Figure 10 is the E2E delay of the ARM-DDS protocol and the SW-ARQ protocol under the comprehensive reliability change of the data stream. The following can be seen from Figs. 10 and 11:

(1) The differentiated data stream which has the different reliability requirements that cannot be met with the SW-ARQ protocol. Therefore, the transmission delay of the protocol does not change by following when the overall reliability of data stream changes.

(2) The overall delay time of the ARM-DDS protocol will be more largely under the higher reliability requirements comprehensively. The reason is that the increase of transmission delay is caused by the need of the node for more retransmission times to ensure the data arrival rate accurately when the comprehensive reliability of the data stream becomes higher.

(3) As can be seen from Fig. 10, the ARM-DDS protocol can reduce the transmission delay time significantly, besides the obvious reduction of the delay was procured by the larger hop count of the node.

(4) The reduction of the ARM-DDS protocol's delay is more obvious while the reliability requirement is high, and when the reliability requirement is low, the reduction of the near-sink node's delay is more obvious. Because the maximum number of 


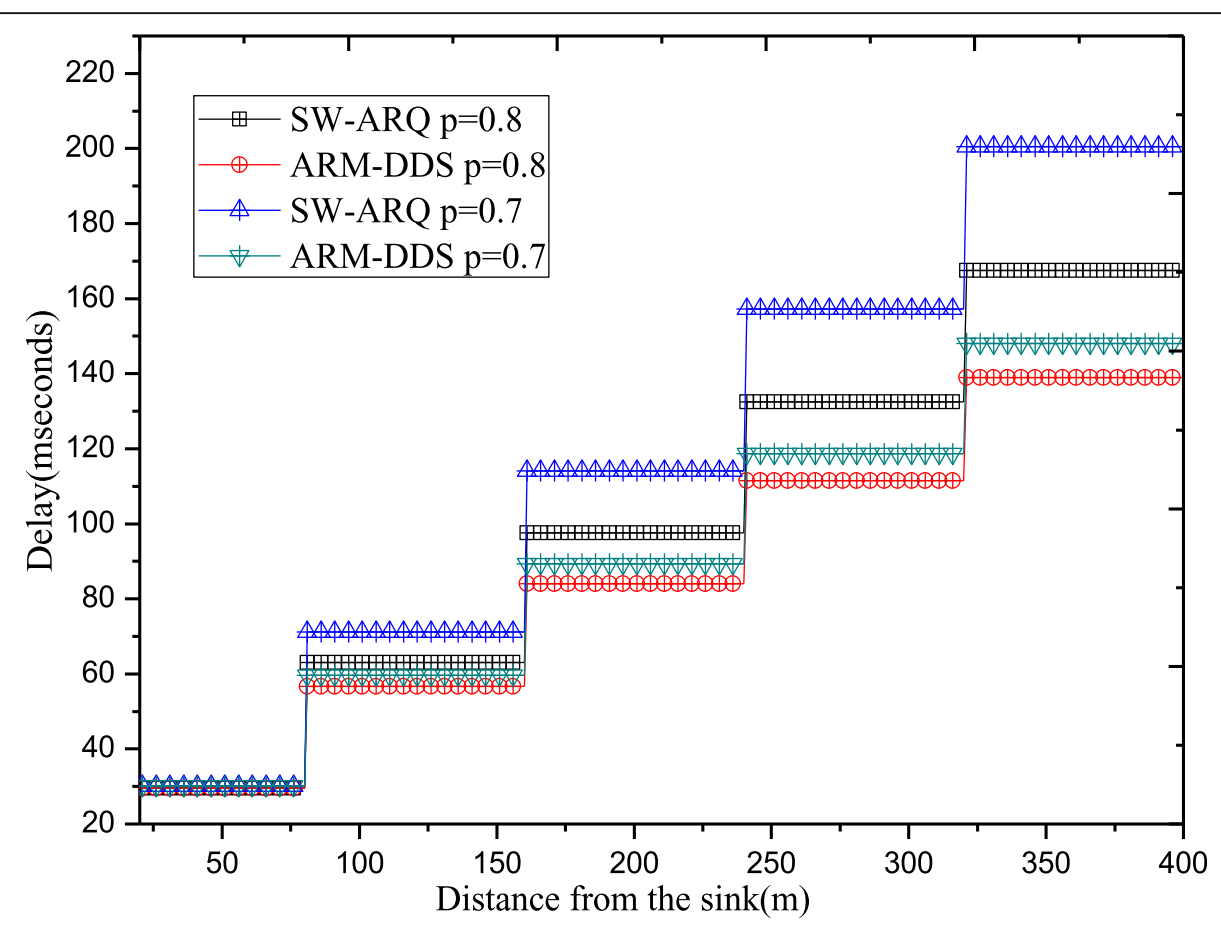

Fig. 8 End-to-end delay comparison with different $p_{i}^{s}$ values

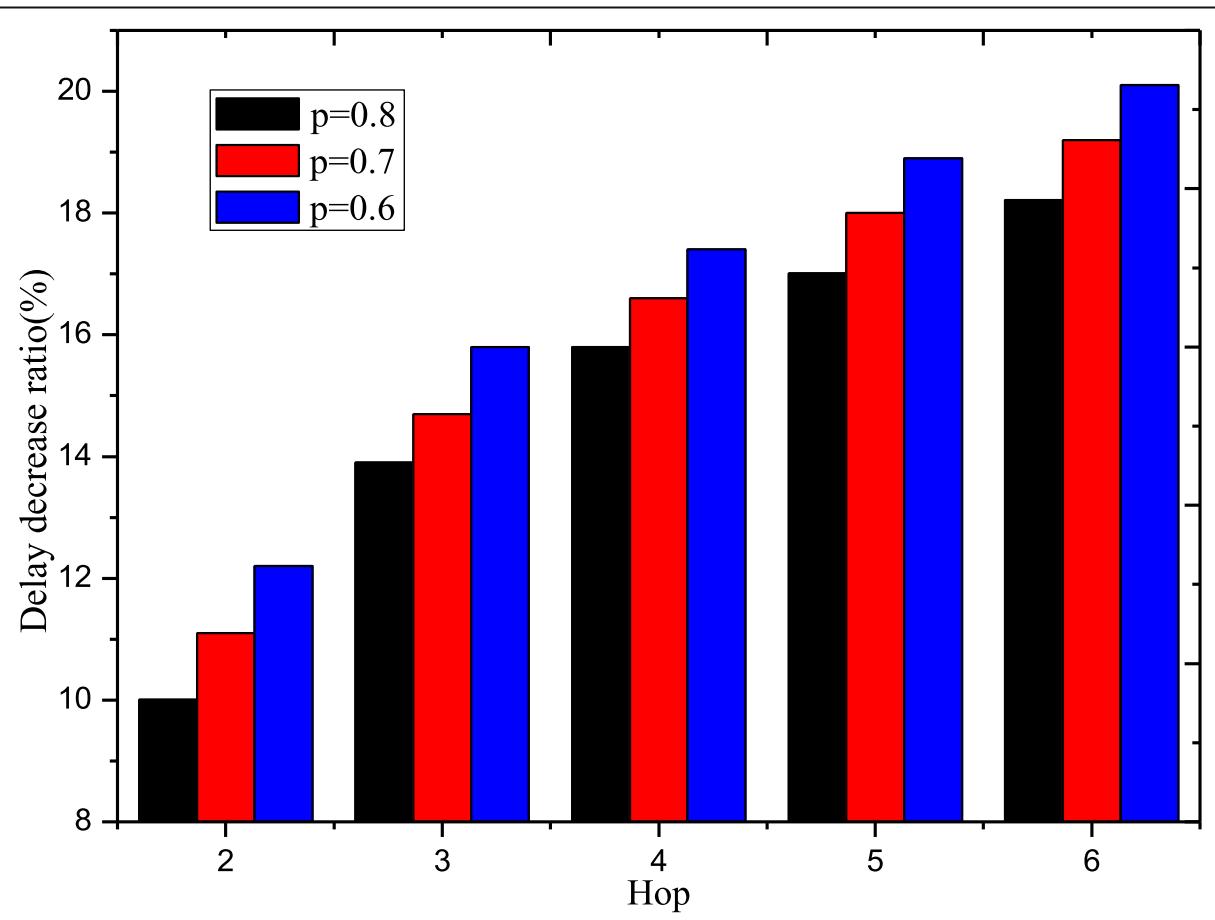

Fig. 9 E2E-weighted delay reduction rate of each hop under different $p_{i}^{s}$ values 


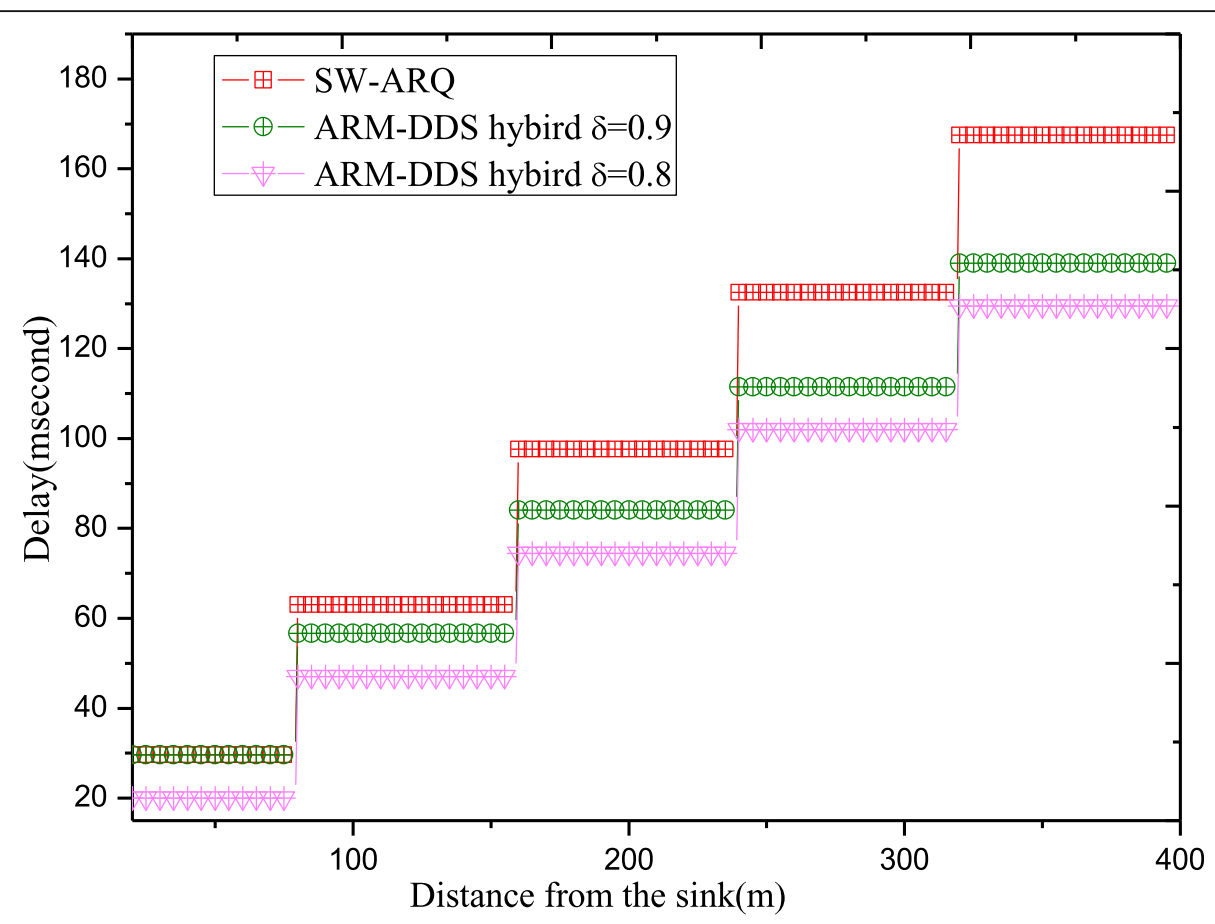

Fig. 10 End-to-end delay comparison under different $\delta$

retransmissions times which near the sink nodes is small, at the same time, ARM-DDS protocol transmits $c$ packets when it nears the sink, which makes it easier to meet the data arrival rate under highreliability requirements.
However, when the reliability requirement is low, the $c$ value near the sink is close to 1 , and the delay reduction rate becomes smaller.

Figure 12 illustrates the average weighted delay of the ARM-DDS protocol and the SW-ARQ protocol for

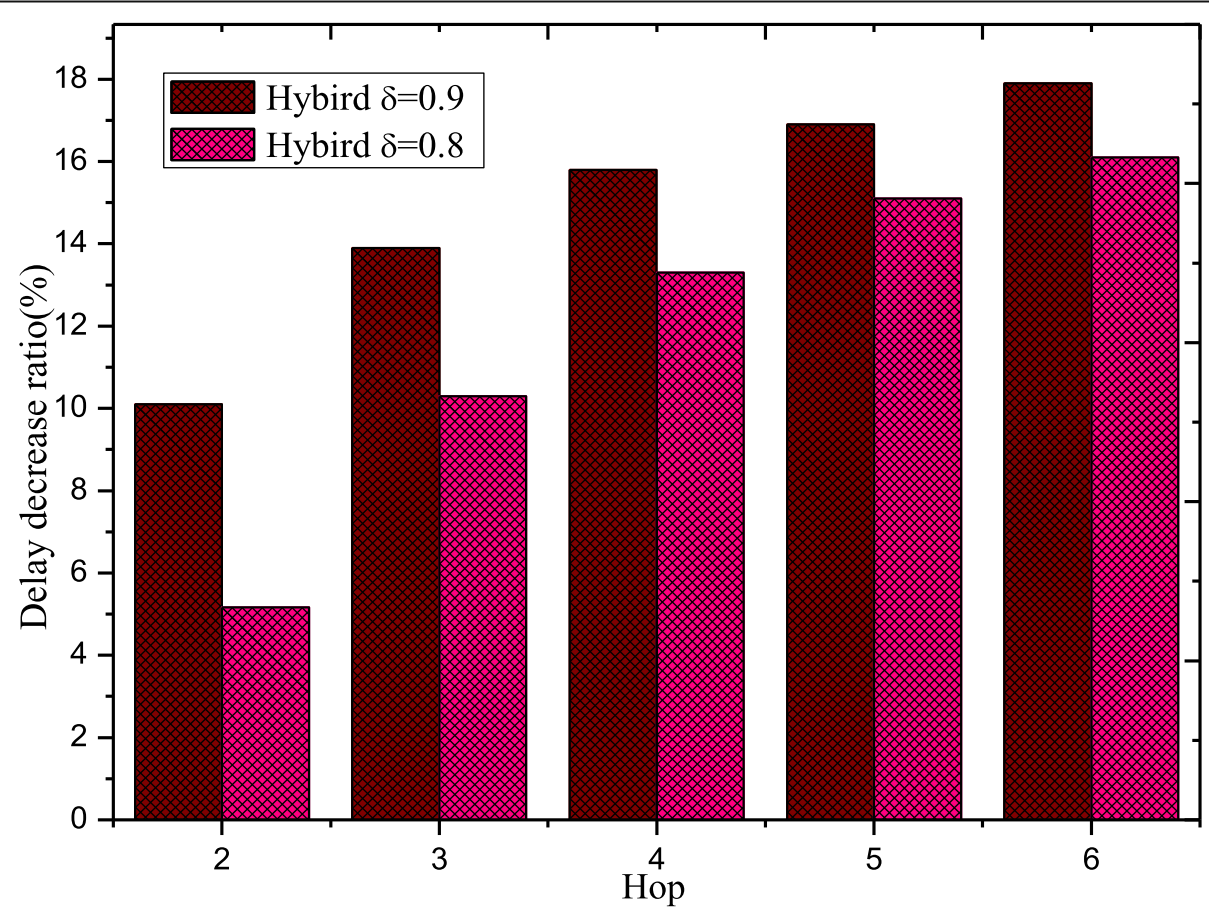

Fig. 11 E2E-weighted delay reduction rate under different $\delta$ 


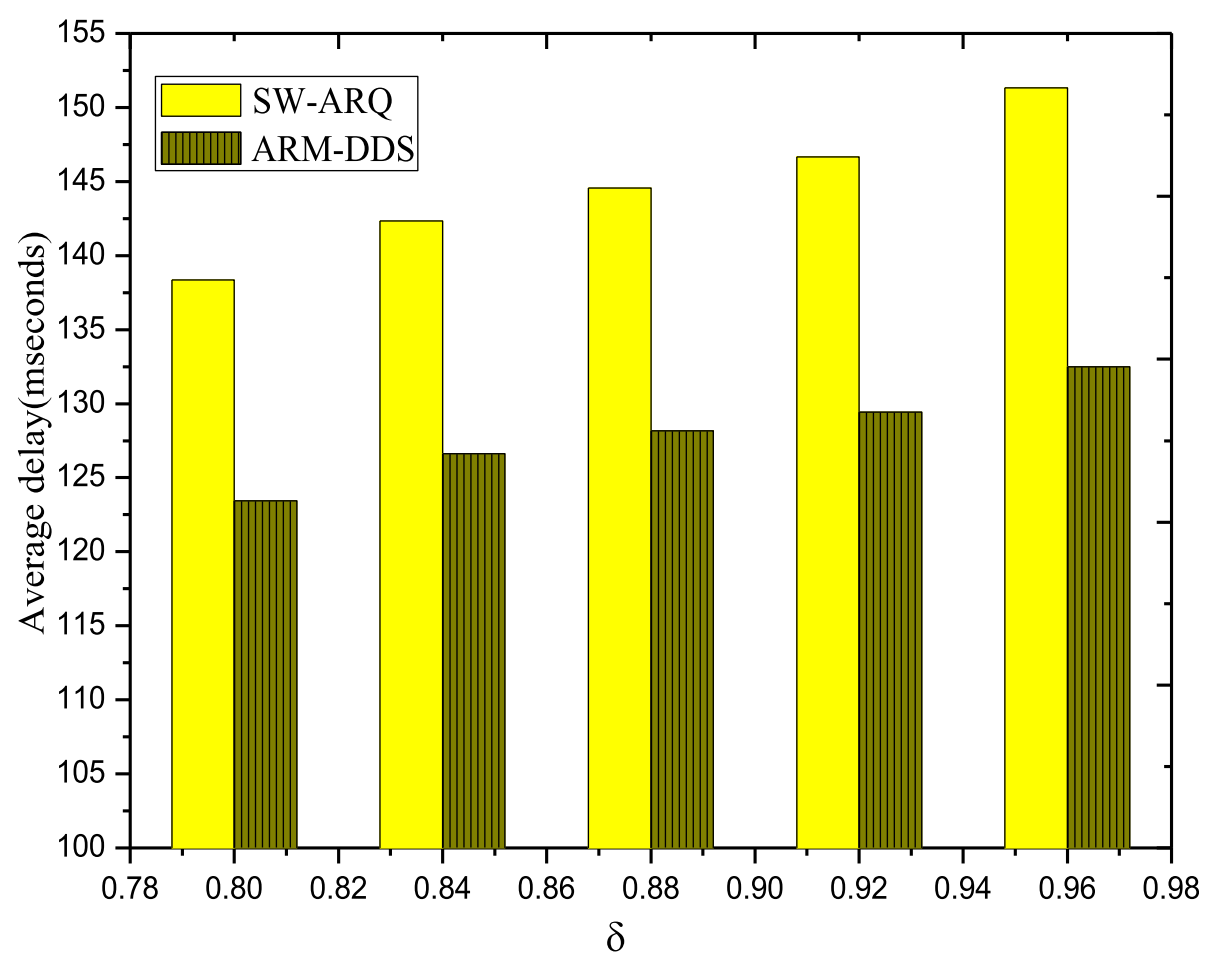

Fig. 12 Comparison of average weighted delays under different $\delta$

different reliability requirements. It can be seen from Fig. 12:

(1) With the following of reliability increasing, the average weighted delay of both protocols reveals the upward trends. Furthermore, in order to meet the higher reliability, the number of retransmissions which expected by the node is increased, and it obviously causes the increasing of the average weighted delay.

(2) In terms of average weighted delay, the traditional SW-ARQ protocol is superlative than the ARMDDS protocol because of the SW-ARQ protocol delay speed of almost $145 \mathrm{~ms}$, while the ARM-DDS can reduce the delay to $127 \mathrm{~ms}$. There are some explanations about the situations of both protocols. The ARM-DDS protocol uses $c$-group data to transmit at the same time; it only needs to return an ACK once, which greatly reduces the delay of ACK return. In the case that the traditional SW-ARQ protocol costs many times to return the ACKs under the lower transmission success rate situations. But as for the ARM-DDS protocol, it can reduce the delay which is caused by saving as part of return time. Although the ARM-DDS protocol can reduce latency in this case, its advantages are not obviously in the case of good network conditions in comparison with the SW-ARQ protocol.
Figure 13 shows the delay reduction rate of the ARMDDS protocol and the SW-ARQ protocol under the different reliability requirements $\delta$. It is obviously to optimize the transmission delay when the reliability $\delta$ is between 0.80 and 0.96 , the ARM-DDS protocol can reduce the delay by $12-14 \%$ in compare with the traditional SW-ARQ protocol. As the reliability requirements increase, the rate of delay reduction increases. Explain that the ARM-DDS strategy performs better under high-reliability requirements.

Thus, the ARM-DDS protocol can be used to transport data streams that require more timeliness.

Figures 14 and 15 show the delay comparison of the ARM-DDS protocol with the traditional SW-ARQ protocol under different $\tau_{\text {round }}$. The following conclusions can be drawn:

(1) $\tau_{\text {round }}$ has a great influence on a data transmission delay. When $\tau_{\text {round }}$ increases, the end-to-end delay of each node will increase significantly.

(2) The larger the $\tau_{\text {round }}$, the more significant the effect on the ARM-DDS protocol to reduce the delay. Because in the traditional SW-ARQ proto$\mathrm{col}$, the periods during the node which send a data packet and prepare to send the second data packet named as $\tau_{\text {round }}$. According to Fig. 3 . The node under the ARM-DDS protocol continuously transmits $c$ data packets; it takes $c(k-1)+\frac{1}{2} \tau_{\text {round }}$ time 


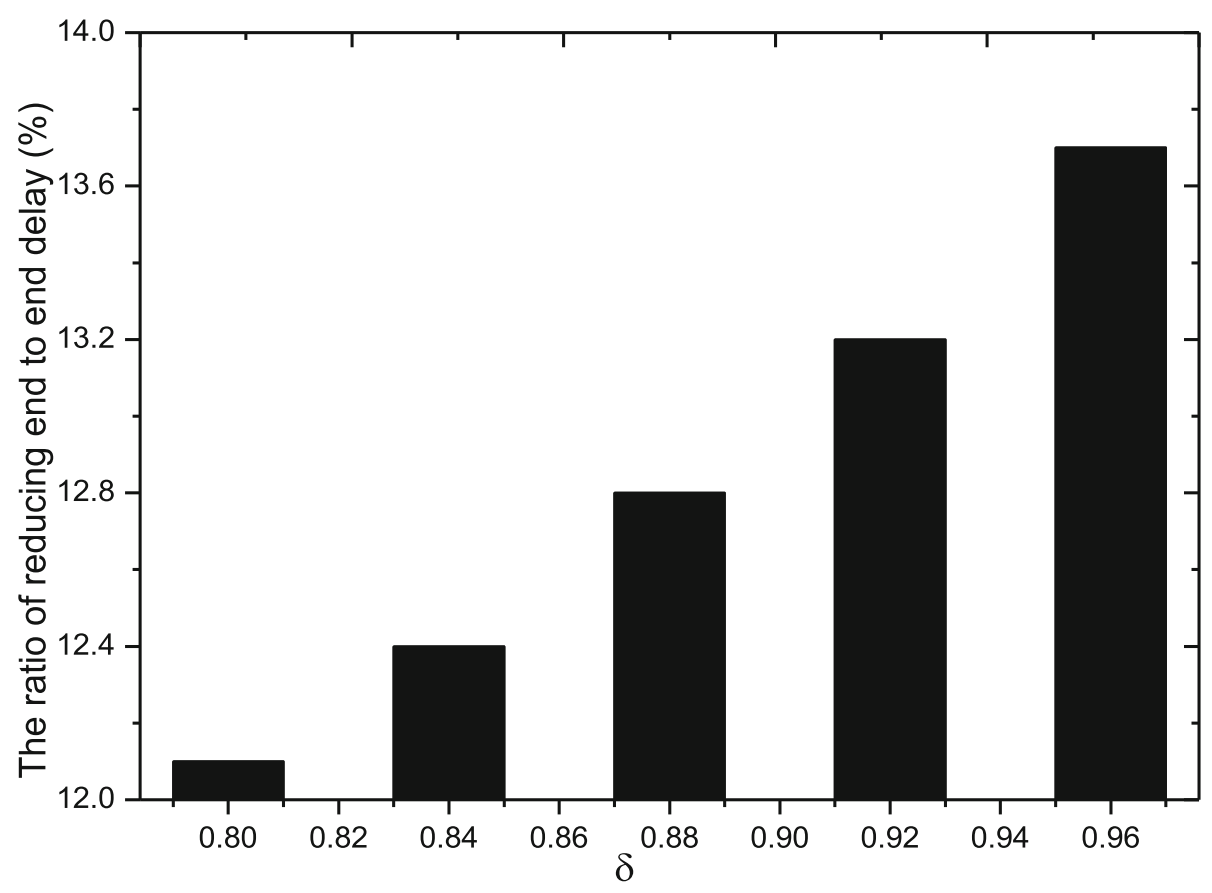

Fig. 13 The average delay reduction rate of ARM-DDS under different $\delta$

and only cost the $c(k-1)+\tau_{\text {round }}$ which adds the extra time of receiving an ACK.

However, if SW-ARQ sends $c$ packets, it will consume the time associated with $\mathrm{c} \tau_{\text {round }}$. So when $\tau_{\text {round }}$ increases, the delay of SW-ARQ is bigger than that of ARM-DDS.
Figure 16 is intended to examine the delay reduction of the ARM-DDS protocol that is influenced by the different transmission radii $r$. The conclusion is given by the information from Fig. 16: With the transmission radius changes, it causes the number of hops of the node changes as following. But the delay did not change

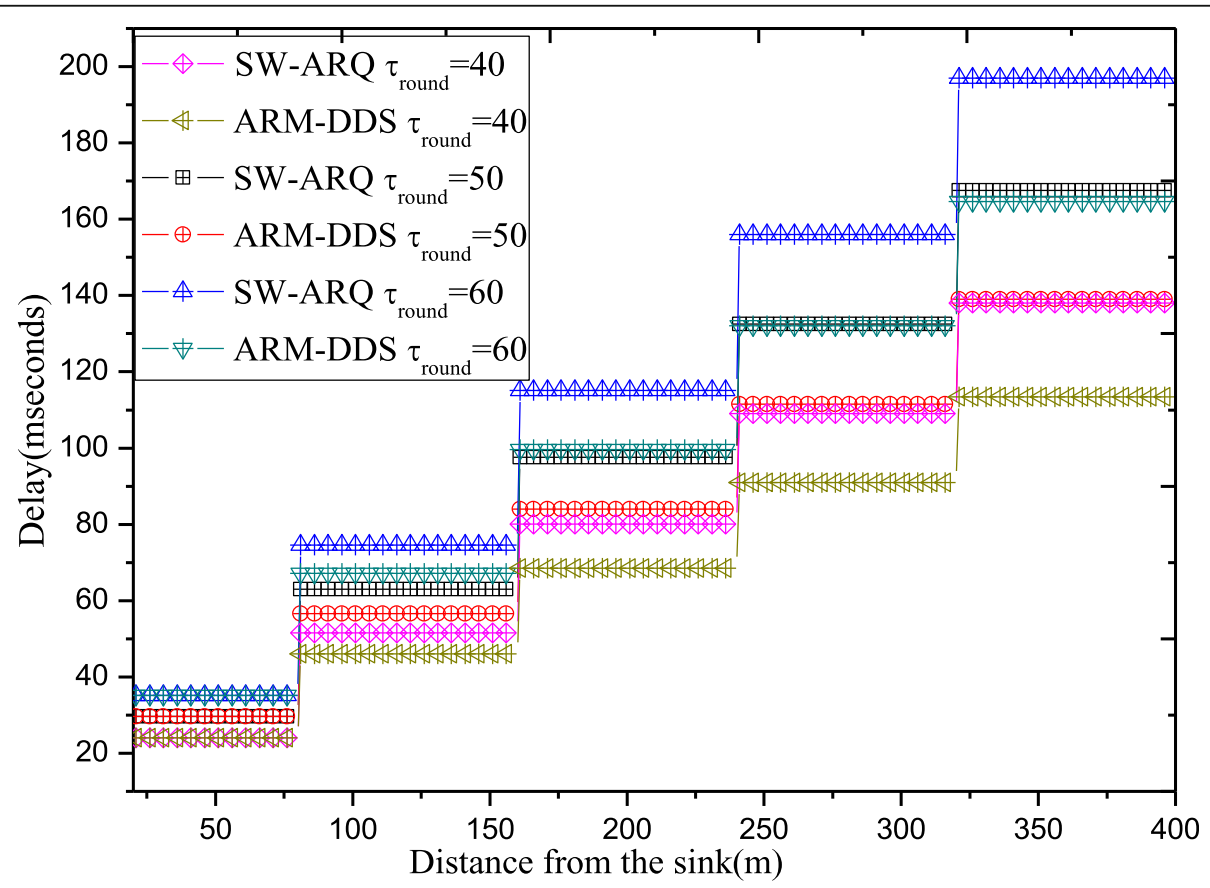

Fig. 14 End-to-end delay comparison under different $\tau_{\text {round }}$ 


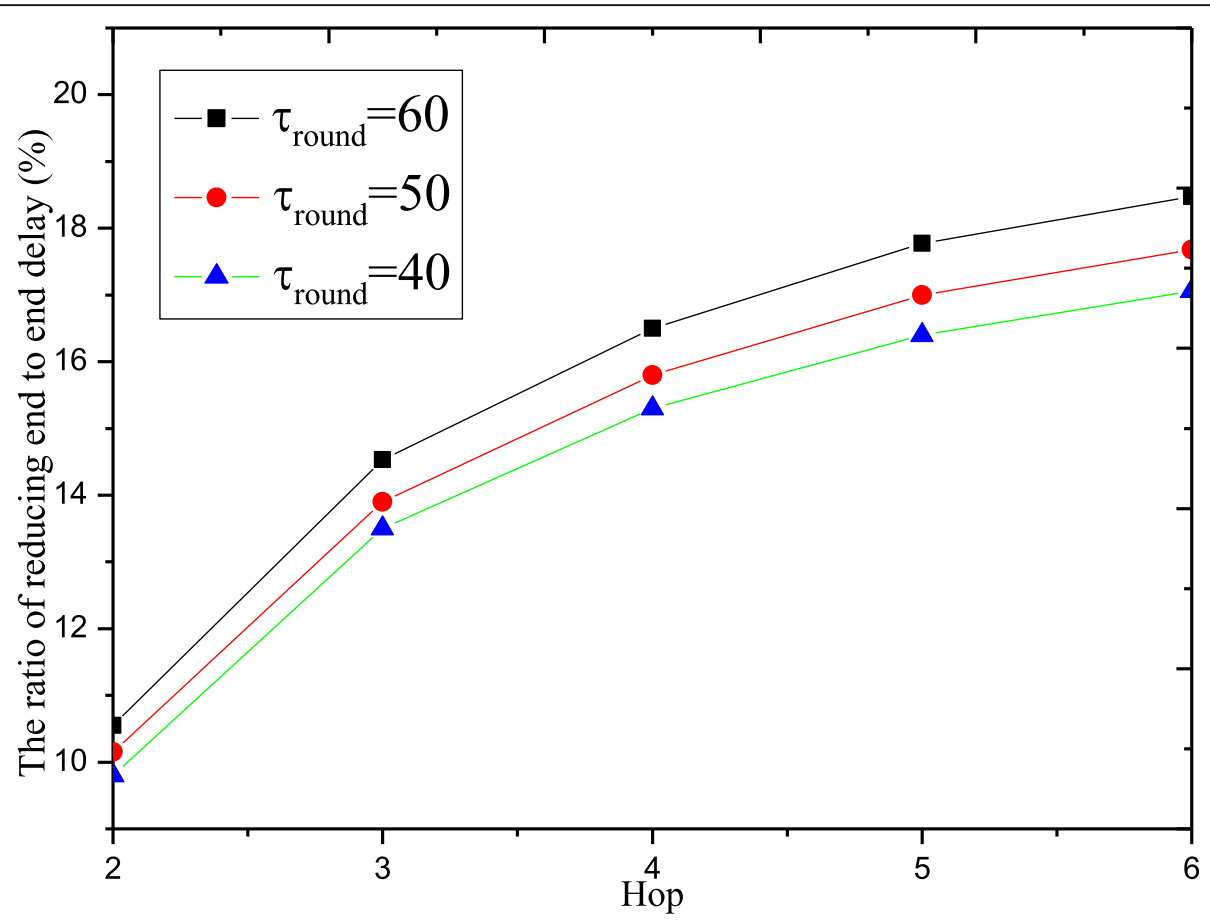

Fig. 15 Decrease rate of each hop under different $\tau_{\text {round }}$

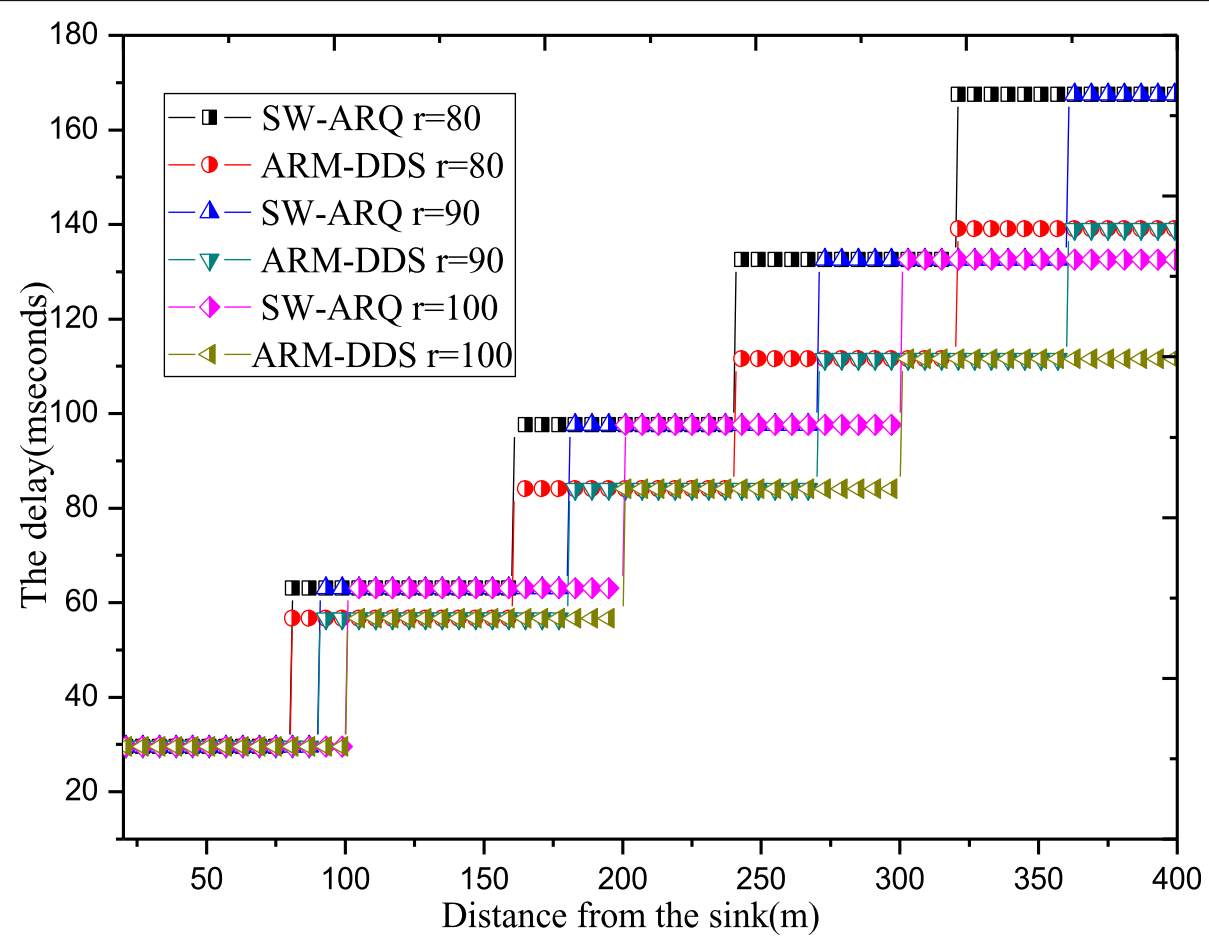

Fig. 16 Comparison of data received by nodes under different emission radii 
significantly. So we conclude that the variation of the transmission radius does not significantly affect the ARM-DDS reduction delay.

\subsection{Energy consumption comparison}

Figures 17, 18, and 19 show the results of the comparison of data reception and transmission amount between the legacy protocol and the ARM-DDS protocol. The information can be obtained from Figs. 17, 18, and 19:

(1) When the ARM-DDS protocol which is near the sink node has been adopted, its transmission data amount is smaller than the SW-ARQ protocol. Because in the differentiated service of the ARM-DDS protocol, each data stream sets the number of transmissions depends on its reliability requirements. However, the SW-ARQ protocol selects the data stream with the highest reliability requirement to set the number of retransmissions of all data packets. At that moment, the amount of transmitted data near the sink of the ARM-DDS protocol will be smaller than the SWARQ protocol which is caused by the number of retransmissions of the ARM-DDS protocol is smaller than the conventional protocol.

(2) To make the comparison of receiving data volume of the two protocols, the data reception amount of the ARM-DDS protocol is larger than the SW-ARQ protocol. Because the data is transmitted using the
ARM-DDS protocol at the far-sink nodes, the amount of reception will increase due to the number of times the node sends will be greater than the maximum number of retransmissions.

(3) As long distances between a node to sink, the amount of data transmitted and received by the ARM-DDS protocol is dramatically improving in comparison with the SW-ARQ protocol.

Hence, it is found that the energy consumption of ARM-DDS which is far from the sink protocol is larger than the energy consumption of the SW-ARQ protocol, and the ARM-DDS protocol could effectively improve the energy usage rate of the network.

Figure 20 emphasis that the maximum number of $c$ value retransmissions times of the ARM-DDS protocol interactive with the SW-ARQ protocol under different $p_{i}^{s}$. As shown in Fig. 20: With the ARM-DDS protocol reducing the delay as much as possible, its $c$ value is under the maximum number of retransmissions. The ARM-DDS protocol does not affect the network lifetime due to the value of $c$ selected by the near-sink node that does not exceed the maximum number of retransmissions. On the other hand, the ARM-DDS protocol can increase the node energy consumption in the far-sink area. Thus, the ARM-DDS protocol can increase the energy utilization of the entire network without reducing network lifetime.

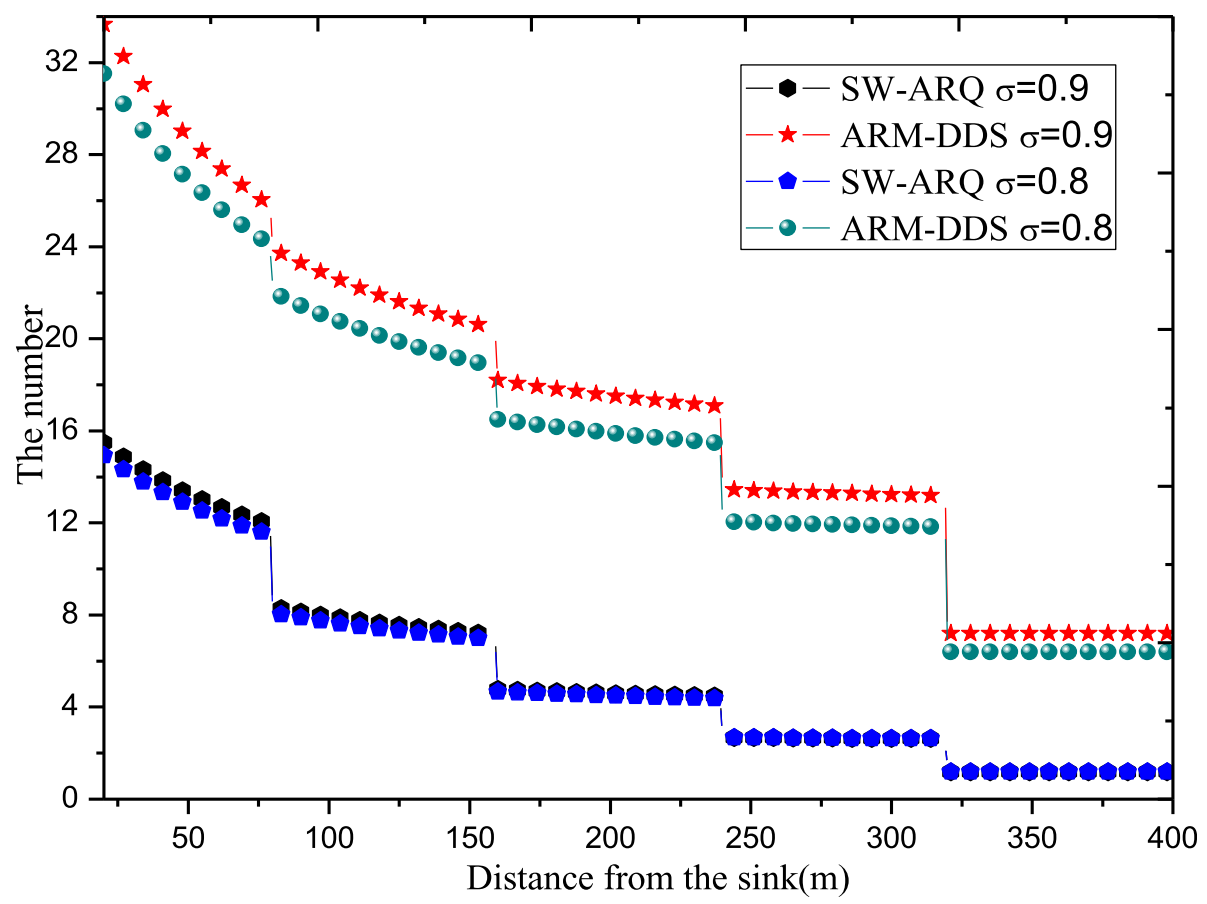

Fig. 17 Comparison of data received by nodes under different $\delta$ 


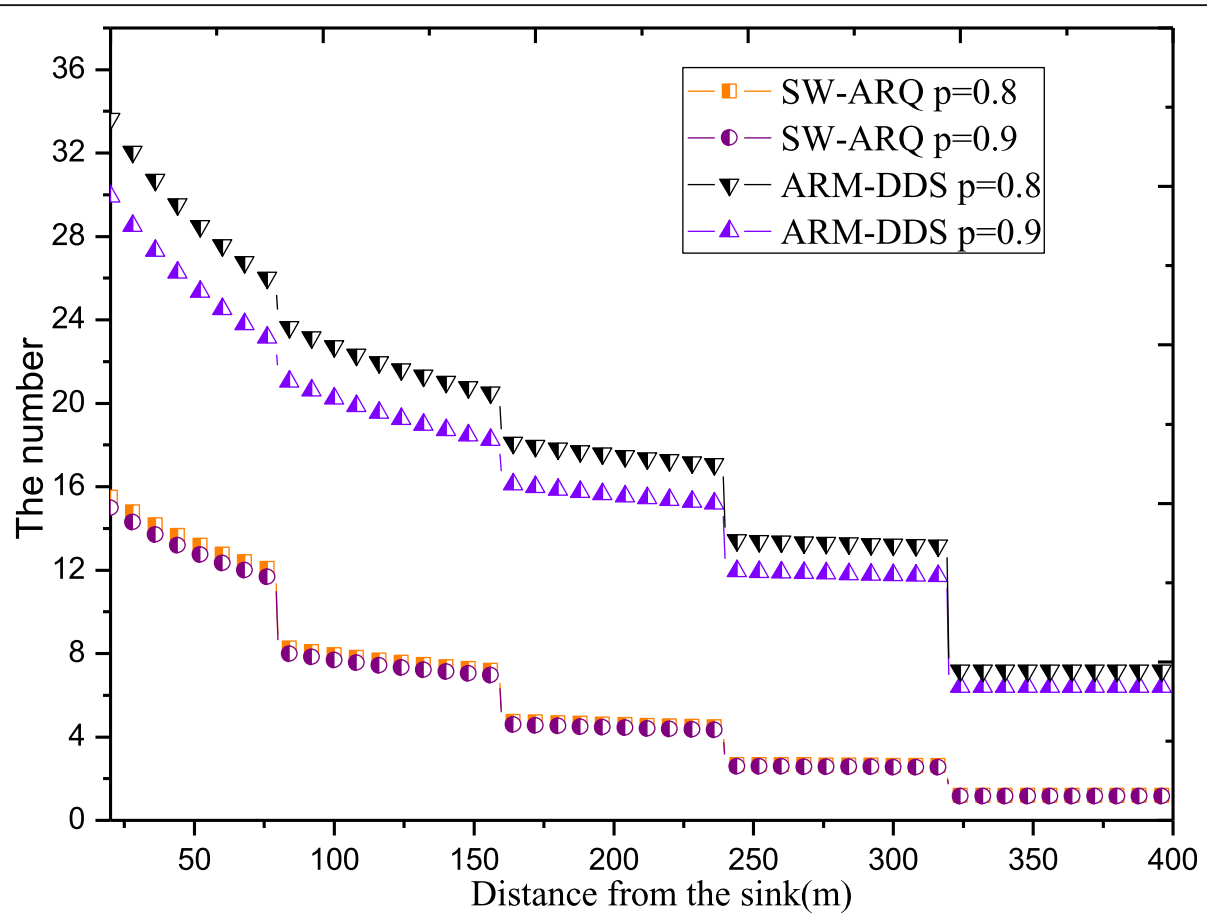

Fig. 18 Different $p_{i}^{s}$ nodes send data volume and ACK comparison

From the system model given in Section 4, the network lifetime is defined as the death time of the first node in the network. In a flat network, the nodes near the sink not only need to undertake the energy of data transmitting that was created by themselves but also the amount of data transferred from the far-sink nodes due to the "many to one" data collective models throughout the centralization of the sink. Hence, the node near the sink will have the largest energy consumption in the entire network. So the first dead node in the network is

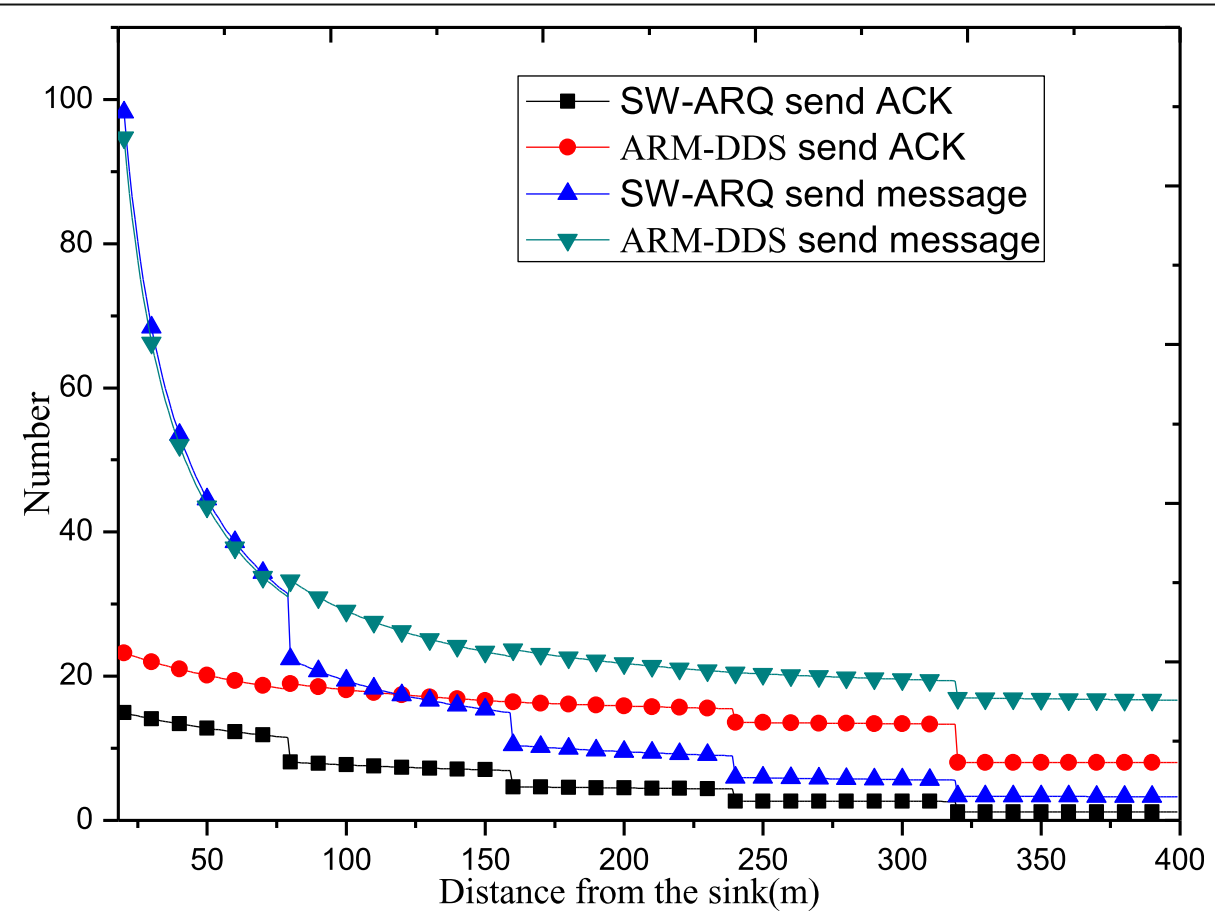

Fig. 19 Node sends data volume and ACK comparison 


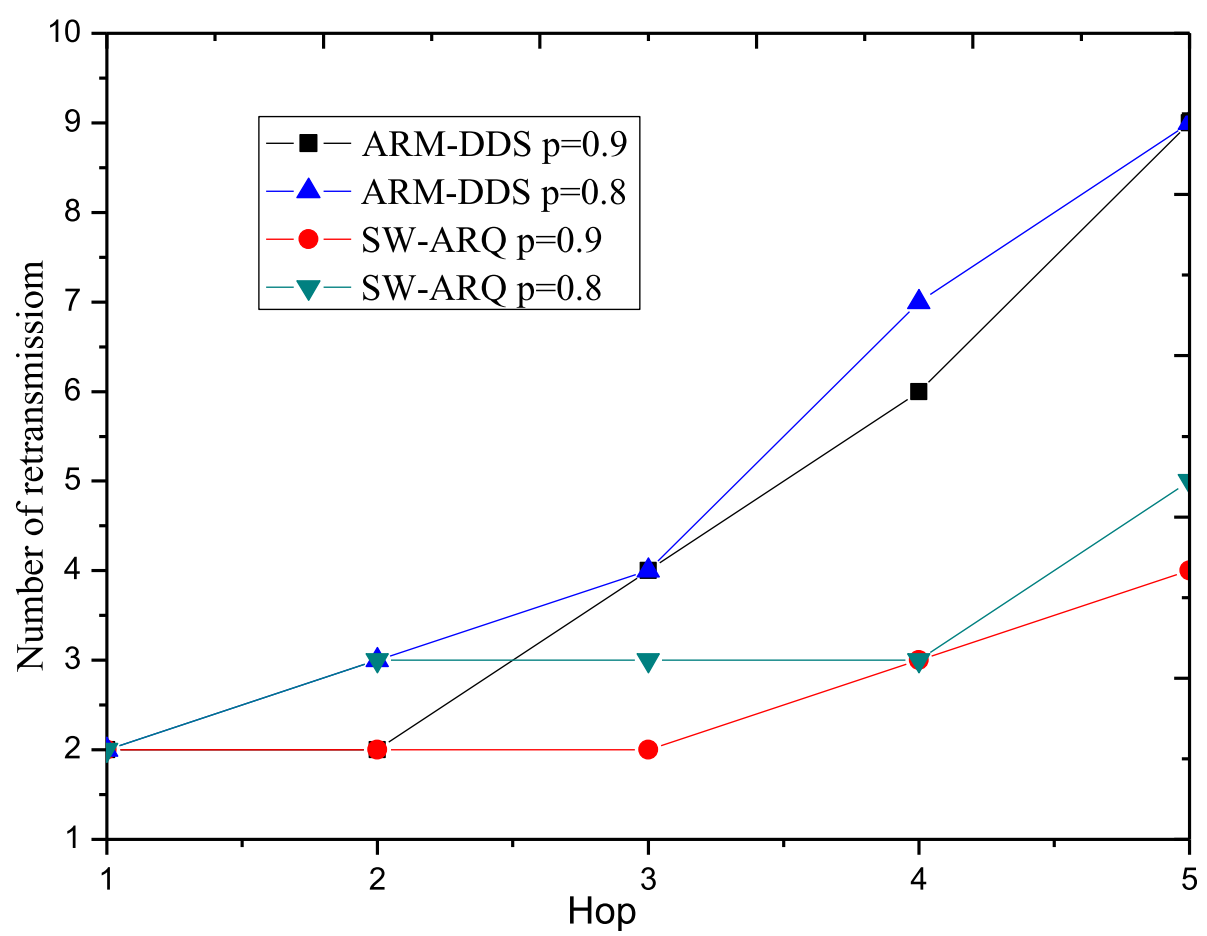

Fig. 20 Comparison of data optimal retransmission times under different $p_{i}^{s}$

usually one of the nodes close to sink. Figures 21 and 22 show the total energy consumption of a node in one cycle according to Theorem 2. Figures 21 is a comparison diagram of the SW-ARQ protocol and ARM-DDS energy consumption. The conclusions are as follows:
(1) The node farther away from the sink which can cost the small energy consumption. This phenomenon also applies to both protocols. Because according to Eq. (5), the farther away from the node, the smaller the amount of data he needs

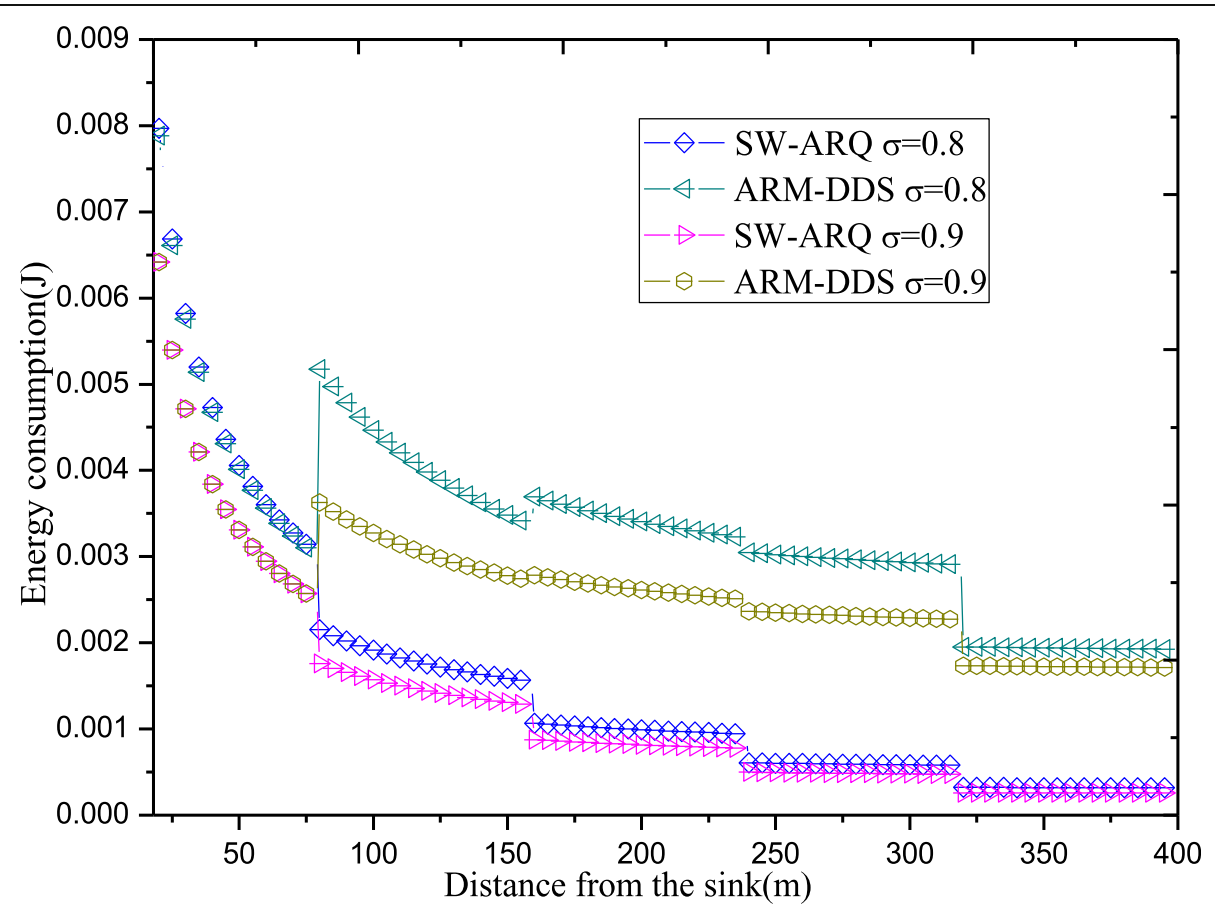

Fig. 21 Comparison of node single wheel energy consumption under different $\delta$ 


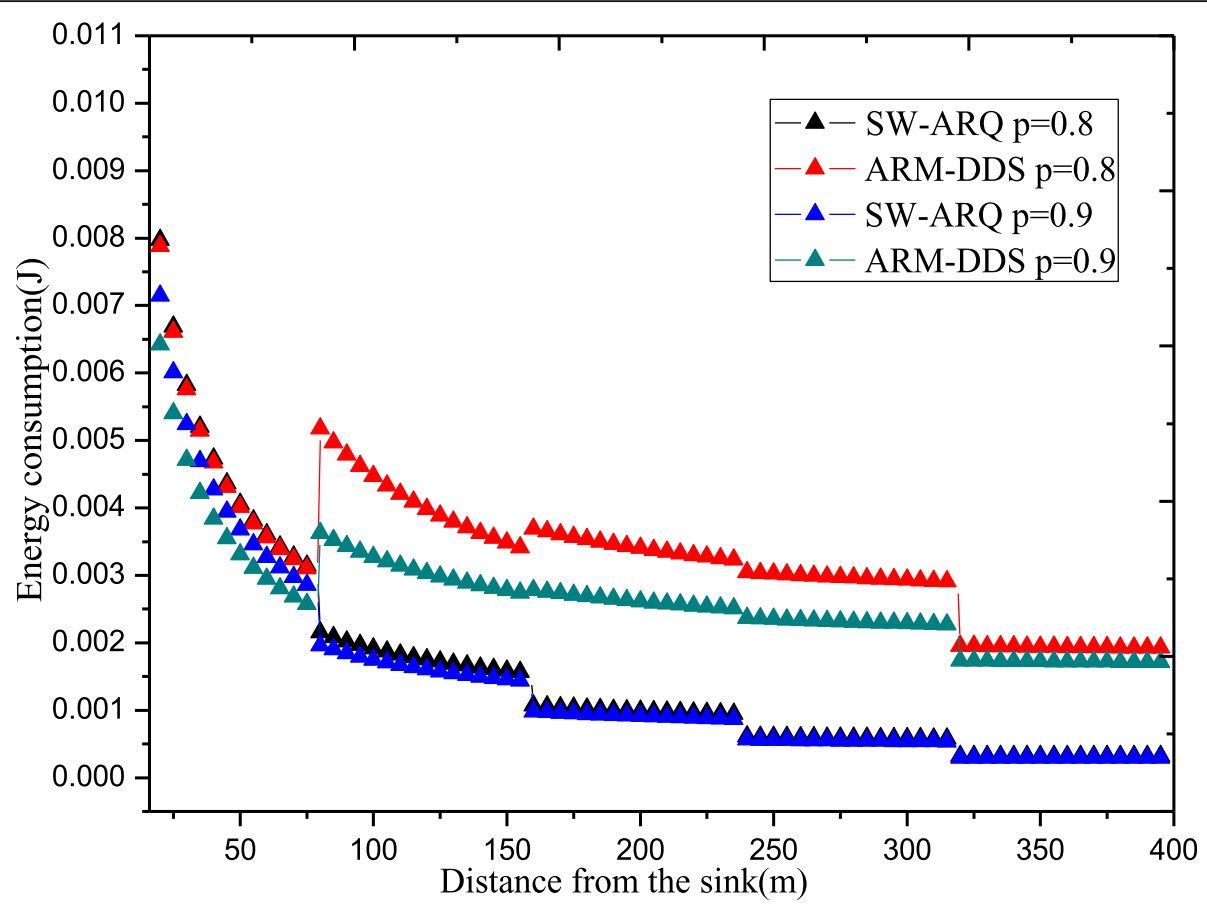

Fig. 22 Comparison of node single wheel energy consumption under different $p_{i}^{s}$

to transmit. So the farther away from the sink, the less energy the node consumes.

(2) The ARM-DDS protocol consumes more energy than the SW-ARQ protocol when studying the energy consumption of nodes at the far-sink. This is because the ARM-DDS transmission scheme continuously sends $c$ packets of data every hop. In contrast, the SW-ARQ protocol only sends one packet at a time. Considering the far-sink node has a lot of residual energy, the $c$ value set by the ARM-DDS protocol will exceed the maximum number of retransmissions. Therefore, the total energy consumption of the nodes of the ARM-DDS protocol is generally higher than the SW-ARQ protocol.

(3) A higher data transmission success rate will make the energy consumption of the node smaller. Because Eq. (7) shows that the larger the transmission success rate $p_{i}^{s}$, the smaller the maximum number of retransmissions of the node. According to Algorithm 2, the value of $c$ of the ARM-DDS protocol will also become smaller.

(4) The energy consumption of the near-sink nodes of the SW-ARQ protocol will be higher. The reason is that the ARM-DDS protocol does not require all data streams to be retransmitted according to the maximum reliability requirements.

Therefore, the SW-ARQ protocol at the near-sink nodes expects the number of retransmissions to be higher than the ARM-DDS protocol. If the network transmission environment deteriorates, the transmission success rate will become lower. As can be seen from the figure, the energy consumption of the ARM-DDS protocol is smaller than the SW-ARQ protocol. And it is more obvious when it is higher than the $p_{i}^{s}$ value.

Figure 23 illustrates the energy utilization rate under different strategies. Energy utilization rate refers to the ratio of the remaining energy of the network to the initial total energy of the network when the network dies. From the experimental results of Fig. 23, the energy utilization rate of ARM-DDS is about $65 \%$, and the energy utilization rate of SW-ARQ is about $37 \%$. It can be seen that the ARM-DDS proposed in this paper significantly improves network energy utilization.

From Fig. 24, it can be shown that: ARM-DDS increases almost $28 \%$ of energy utilization in compare with the SW-ARQ protocol. Moreover, energy utilization has a minimal effect on both the transmission and reliability requirements of the network. All of these indicate that the ARM-DDS protocol proposed in this paper has a positive influence on energy utilization compared to the SW-ARQ protocol.

\section{Network lifetime and reliability optimization} In terms of network life optimization, the ARM-DDS scheme can increase $\frac{\bar{\delta}}{\delta_{\max }} \%$ relative to the SW-ARQ protocol, where $\bar{\delta}$ is the average weighted reliability re- 


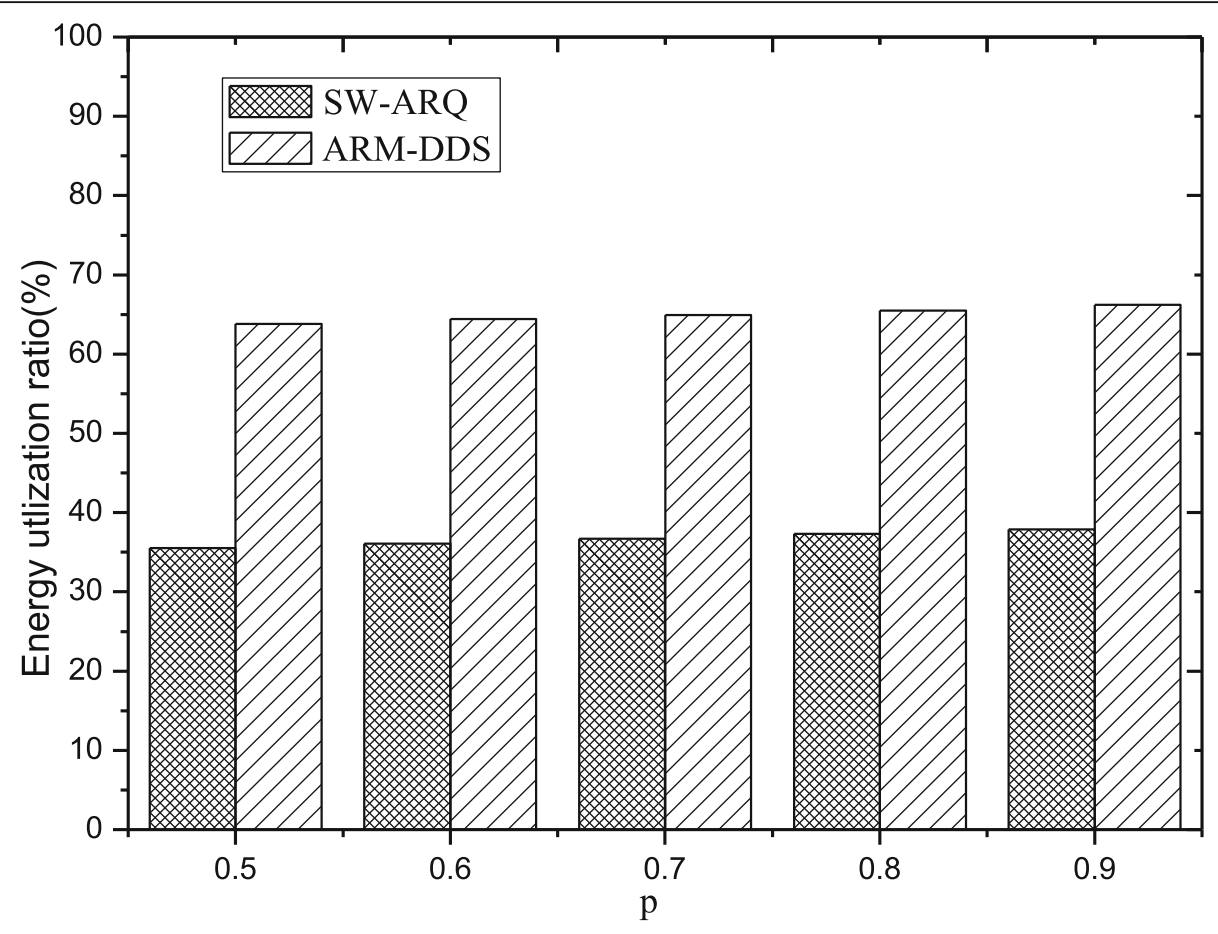

Fig. 23 Energy-efficiency of different protocols

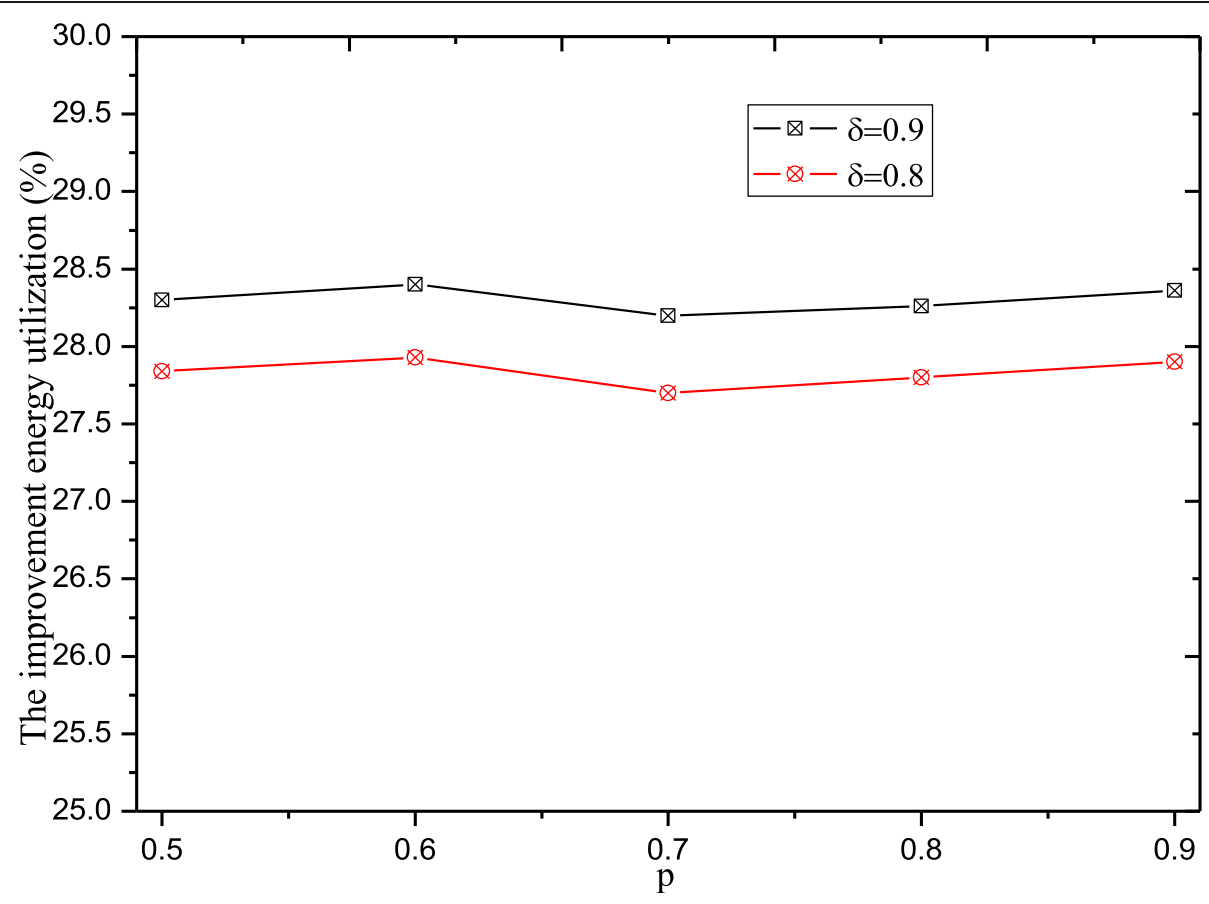

Fig. 24 ARM-DDS increase in energy utilization at different $\delta$ 
quirement for all data streams, and $\delta_{\max }$ is the maximum reliability requirement for all data streams. Figure 25 shows that the situation of network lifetimes for the two protocols under different reliability. The ARM-DDS protocol has a longer lifetime than the SW-ARQ protocol network, because the ARM-DDS protocol adopts the methods of differentiated services. Each data stream sets the value of $c$ according to the needs of reliability. However, the SW-ARQ protocol is ruled to select the highest reliability requirements and to transfer all of the information in the data stream.

Therefore, the amount of data received and transmitted by the ARM-DDS protocol which near-sink nodes is smaller than the SW-ARQ protocol, which optimizes the lifetime of the network.

Figures 26 and 27 are the maximum numbers of retransmissions and the average number of retransmissions of the SW-ARQ protocol at the hop $=3$ in different network environments. In the ARM-DDS protocol, the $c$ does not exceed when the data transmission with high timeliness requiring the maximum number of retransmissions. Because the delay is guaranteed to be as small as possible, the set of $c$ is not too large. Otherwise, when multiple sets of data are continuously transmitted, the time of data transmission will be accumulated and it still affects the delay. However, the value of $c$ will exceed the maximum number of retransmissions when the data stream transmissions among the rules of timeliness are without high requirement.
Figure 28 shows the transmission reliability under the ARM-DDS protocol and the SW-ARQ protocol. The following can be seen from the figure:

(1) The reliability of the ARM-DDS protocol at the farsink nodes is higher than that of the SW-ARQ protocol. Because the $\mathrm{c}$ value of the ARM-DDS protocol at the far-sink nodes is greater than the maximum number of retransmissions of the SWARQ protocol, therefore, the data reliability of the ARM-DDS protocol at the far-sink node is higher than that under the SW-ARQ protocol. This ensures a high data arrival rate even when the transmission conditions are not very good. In terms of transmission reliability of near-sink nodes, since the ARM-DDS protocol uses a differentiated service algorithm, the weighted reliability is lower than the SW-ARQ protocol. However, the ARM-DDS protocol can still meet the requirements of the system while reducing the overall reliability requirements.

(2) When transmitting the high-reliability requirement data, the SW-ARQ protocol waits for an ACK when sending a data packet, which results in a very small increase in transmission reliability at the far-sink nodes. When it is necessary to transmit very reliable data, the SW-ARQ protocol often fails to meet the requirements. The ARM-DDS protocol only starts to listen for ACKs after continuously transmitting multiple sets of data packets. Such a transmission

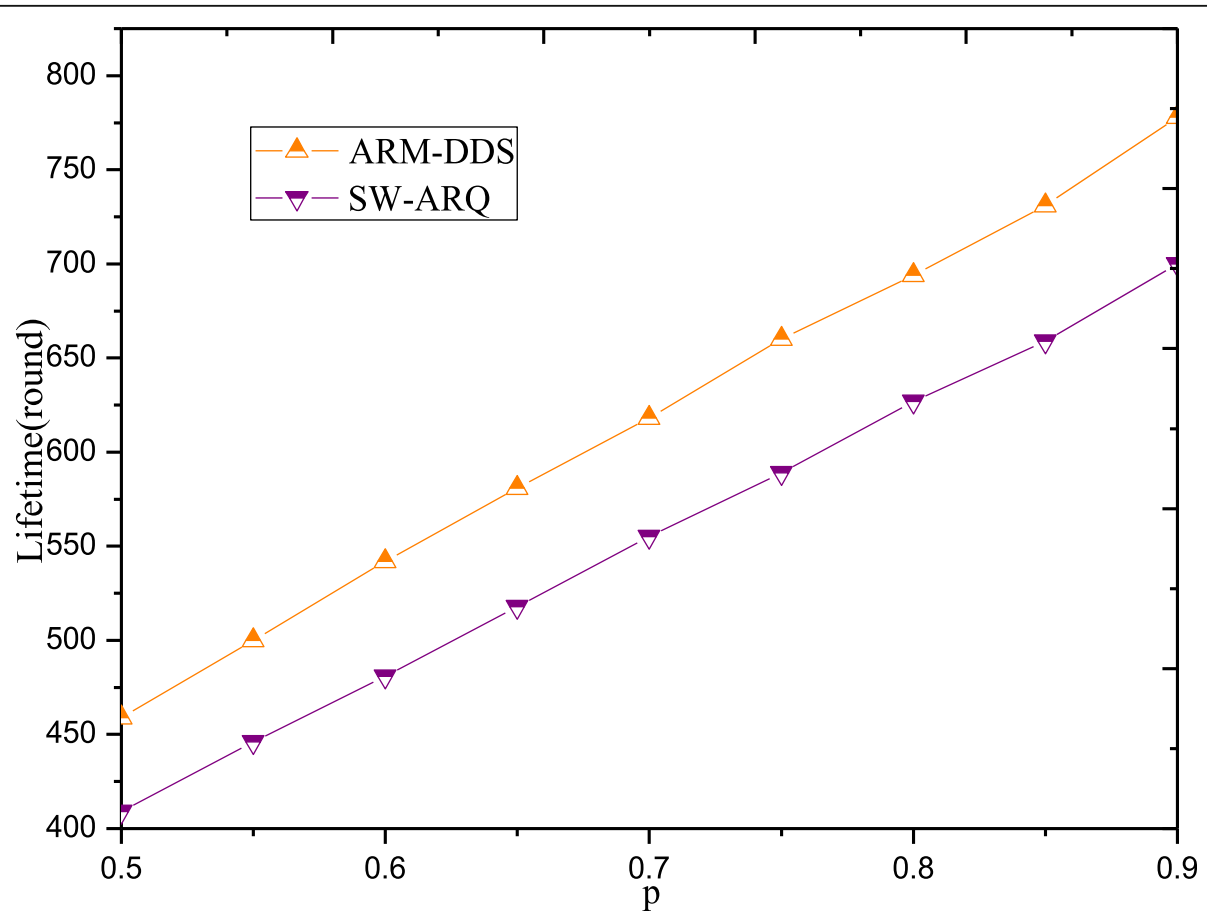

Fig. 25 ARM-DDS can increase network life 


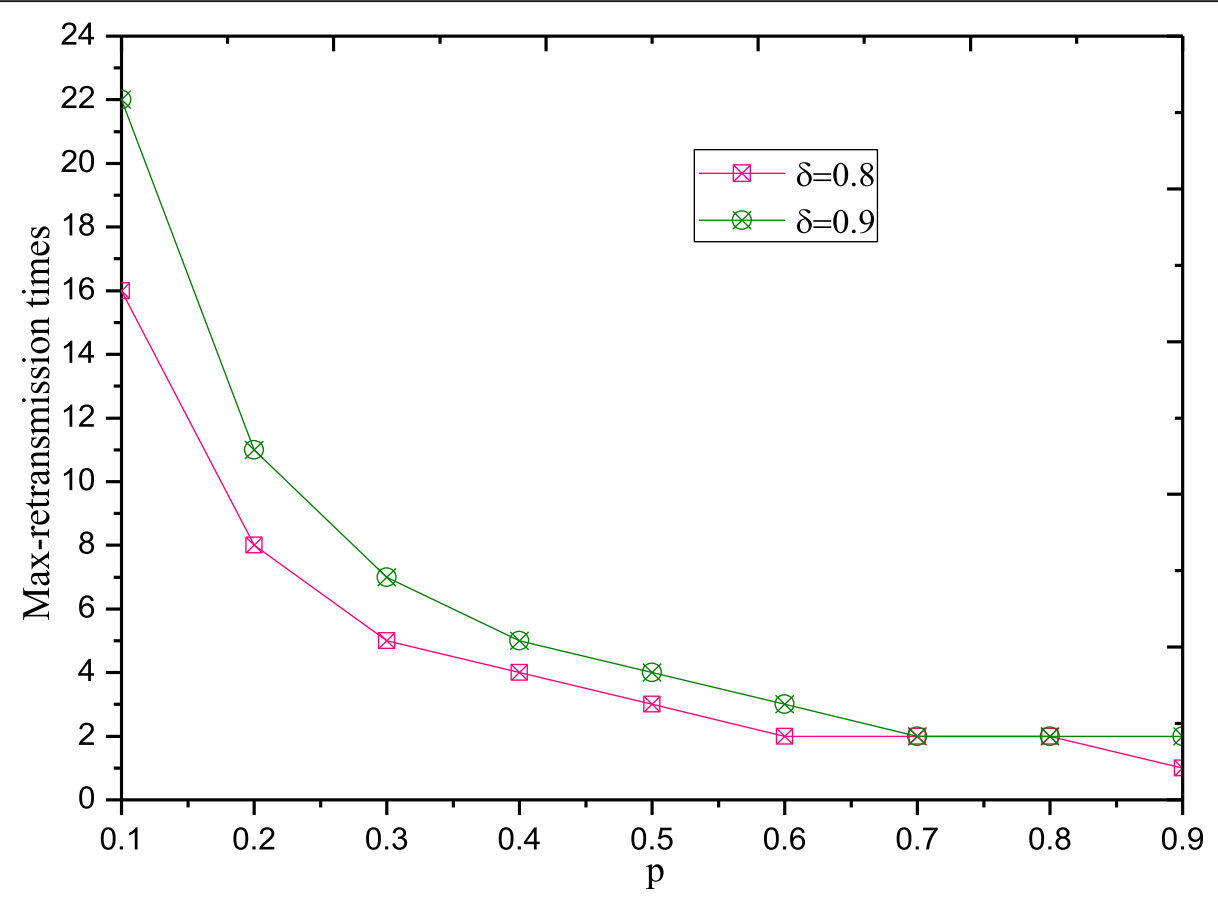

Fig. 26 Maximum number of retransmissions under different $p_{i}^{s}$

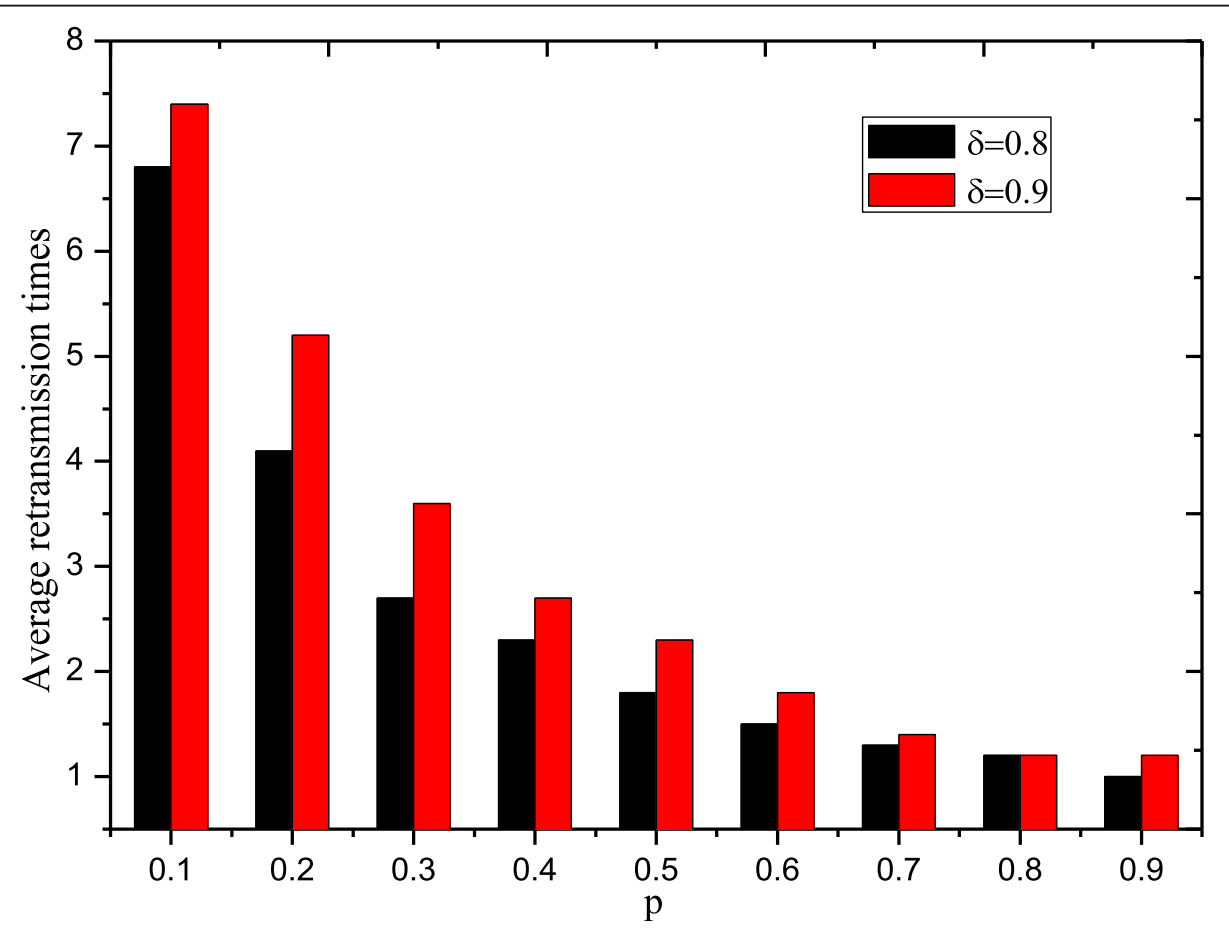

Fig. 27 Average number of retransmissions under different $p_{i}^{s}$ 


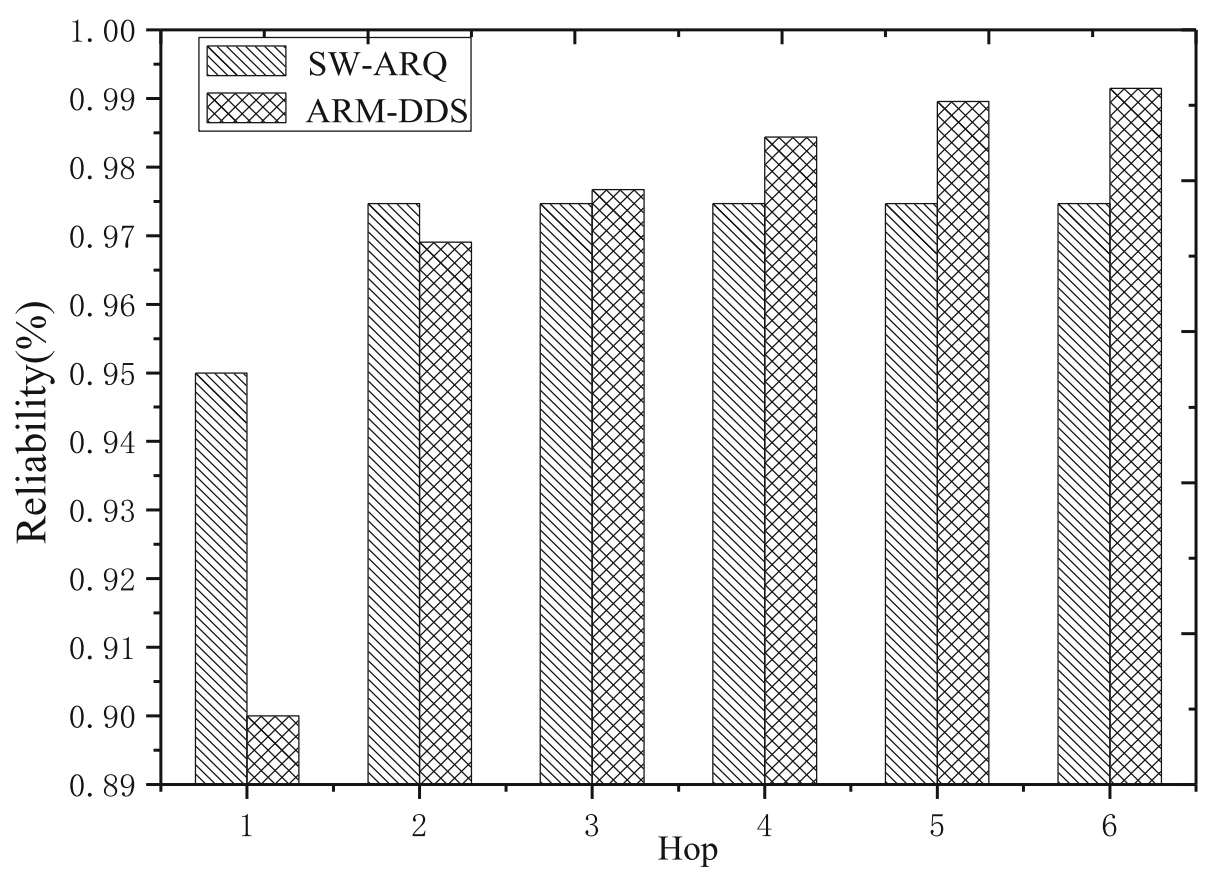

Fig. 28 Comparison of data transmission reliability of different protocols

method greatly increases the upper limit of the number of data packets sent by the system in one transmission period. Therefore, the ARM-DDS protocol can meet the data transmission requirements of higher reliability. Finally, it can be concluded that the ARM-DDS protocol can reduce the number of overall data retransmissions through differentiated services. The ARM-DDS protocol has advantages over the SW-ARQ protocol when transmitting data with high-reliability requirements.

\section{Conclusion}

The most important task in wireless sensor networks is to sense and capture information about the surrounding environment and pass the information back to the sink. Since different applications have different delay and reliability requirements for data, how to effectively differentiate services is important for improving network performance. This paper proposes an Adaptive Retransmit Mechanism for Delay Differentiated Services (ARMDDS) scheme mainly for the research that there is no better delay differentiated service. In the ARM-DDS protocol, the application allocation is faced with different requirements and different transmission methods. Thus, it can meet all application requirements while reducing delays and increasing network lifetime. At the same time, the remaining energy in the network is utilized to increase the energy utilization of the network. This paper tests the performance of the ARM-DDS protocol through theoretical analysis and simulation experiments. The experimental results show that ARMDDS can effectively differentiate the data according to the traditional protocol. In the delay aspect, as long as the E2E delay requirement of the application is not lower than $87.9 \%$ of the SW-ARQ protocol, the protocol can be used for transmission. In terms of energy consumption, the ARM-DDS protocol can improve energy utilization by $28 \%$. Finally, according to the reliability requirements, it increases the network life.

\section{Abbreviations}

ACK: Acknowledge; ARM-DDS: Adaptive Retransmit Mechanism for Delay Differentiated Services; E2E: End to end; GBN: Go-Back-N protocol; HBH: Hop by hop; IDDR: Integrity and delay differentiated routing; loT: Internet of Things; MAC: Media Access Control; QoS: Quality of service; RTO: Time to out; RTT: Round-trip time; SEDR: Security and Energy-efficient Disjoint Route; SNR: Signal-to-noise ratio; SR: Selective repeat protocol; SW-ARQ: Send-andwait automatic repeat-request; WSNs: Wireless sensor networks

\section{Acknowledgement}

The authors thank the person who provided meticulous and valuable suggestions for improving the paper.

\section{Authors' contributions}

YC performed the experiment, analyzed the experiment results, and wrote the manuscript. WL, TW, QD, and HS commented on the manuscript. AL conceived of the work and wrote part of the manuscript. All authors read and approved the final manuscript.

\section{Funding}

This research was funded by the National Natural Science Foundation of China (No. 61772554, No. 61602398, No. 61672447) and the Hunan Provincial National Natural Science Foundation of China (No. 2017JJ3316, No. 2019JJ50592).

Availability of data and materials Not applicable 


\section{Competing interests}

The authors declare that they have no competing interests.

\begin{abstract}
Author details
'School of Computer Science and Engineering, Central South University, Changsha 410083, China. ${ }^{2}$ School of Informatics, Hunan University of Chinese Medicine, Changsha 410208, China. ${ }^{3}$ Department of Computer Science and Technology, Huaqiao University, Xiamen 361021, China. ${ }^{4}$ The Key Laboratory of Hunan Province for Internet of Things and Information Security, Xiangtan University, Xiangtan 411105, China. ${ }^{5}$ College of Information Engineering, Xiangtan University, Xiangtan 411105, China. ${ }^{6}$ Department of Electrical, Computer, Software, and Systems Engineering, Embry-Riddle Aeronautical University, Daytona Beach, FL 32114, USA.
\end{abstract}

Received: 26 July 2019 Accepted: 23 September 2019 Published online: 28 November 2019

\section{References}

1. S. Sarkar, S. Chatterjee, S. Misra, Assessment of the suitability of fog computing in the context of Internet of Things. IEEE Transactions on Cloud Computing 6(1), 46-59 (2018)

2. Y. Deng, H. Hu, N. Xiong, W. Xiong, L. Liu, A general hybrid model for chaos robust synchronization and degradation reduction. Inf. Sci. 305(39), 146-164 (2015)

3. X. Xiang, W. Liu, T. Wang, M. Xie, X. Li, H. Song, A. Liu, G. Zhang. Delay and energy efficient data collection scheme based matrix filling theory for dynamic traffic IOT. EURASIP Journal on Wireless Communications and Networking, 168 (2019). DOI: 10.1186/s13638-019-1490-5.

4. L. Hu, A. Liu, M Xie, T Wang. UAVs joint vehicles as data mules for fast codes dissemination for edge networking in smart city. Peer-to-Peer Networking and Applications, (2019). DOl: https://doi.org/10.1007/s12083-019-00752-0.

5. Y. Liu, A. Liu, T. Wang, X. Liu, N. Xiong, An intelligent incentive mechanism for coverage of data collection in cognitive Internet of Things. Futur. Gener. Comput. Syst. 100, 701-714 (2019)

6. X. Sun, W. Liu, T. Wang, Q. Deng, A. Liu, N. Xiong, S. Zhang, Two-hop neighborhood information joint double broadcast radius for effective code dissemination in WSNs. IEEE Access 7(1), 88547-88569 (2019)

7. Z. Wang, T. Li, N. Xiong, Y Pan. A novel dynamic network data replication scheme based on historical access record and proactive deletion. The Journal of Supercomputing, 62 (1), 227-250 (2012)

8. X. Xiang, W. Liu, A. Liu, N. Xiong, Z. Zeng, Z. Cai. Adaptive duty cycle control based opportunistic routing scheme to reduce delay in cyber physical systems. International Journal of Distributed Sensor Networks. 15 (4), Dol: https://doi.org/10.1177/1550147719841870 2019

9. T. Shu, W. Liu, T. Wang, M. Zhao, N. Xiong, M. Ma, X. Li, A. Liu, Broadcast based code dissemination scheme for duty cycle based wireless sensor networks. IEEE Access 7(1), 105258-105286 (2019)

10. Y. Liu, M. Ma, X. Liu, N. Xiong, A. Liu, Y. Zhu. Design and analysis of probing route to defense sink-hole attacks for Internet of Things security. IEEE Transactions on Network Science and Engineering, (2018). DOl: https://doi. org/10.1109/TNSE.2018.2881152.

11. T. Wang, L. Qiu, G. Xu, A. Sangaiah, A. Liu. Energy-efficient and trustworthy data collection protocol based on mobile fog computing in Internet of Things. IEEE Transactions on Industrial Informatics, (2019). DOl: https://doi. org/10.1109/TII.2019.2920277.

12. W. Shi, W. Liu, T. Wang, Z. Zeng, G. Zhi. Adding duty cycle only in connected dominating sets for energy efficient and fast data collection, IEEE Access, (2019) Dol: https://doi.org/10.1109/ACCESS.2019.2937626.

13. M. Huang, W. Liu, T. Wang, Q. Deng, A. Liu, M. Xie, M. Ma, G. Zhang. A game-based economic model for price decision making in cyberphysical-social systems, IEEE Access, (2019) Dol: https://doi.org/10.1109/ ACCESS.2019.2934515.

14. X. Deng, G. Li, M. Dong, K Ota. Finding overlapping communities based on Markov chain and link clustering. Peer-to-Peer Networking and Applications, 10(2), 411-420 (2017).

15. J. Ren, Y. Zhang, N. Zhang, D. Zhang, X. Shen, Dynamic channel access to improve energy efficiency in cognitive radio sensor networks. IEEE Trans. Wirel. Commun. 15(5), 3143-3156 (2016)

16. J. Zhang, F. Ren, S. Gao, H. Yang, et al., Dynamic routing for data integrity and delay differentiated services in wireless sensor networks. IEEE Trans. Mob. Comput. 14(2), 328-343 (2014)
17. Q. Wang, W. Liu, T. Wang, M. Zhao, X. Li, M. Xie, M. Ma, G. Zhang, A. Liu, Reducing delay and maximizing lifetime for wireless sensor networks with dynamic traffic patterns. IEEE Access 7(1), 70212-70236 (2019)

18. Q. Li, A. Liu, T. Wang, M. Xie, N. Xiong, Pipeline slot based fast rerouting scheme for delay optimization in duty cycle based M2M communications. Peer-to-Peer Networking and Applications. (2019). https://doi.org/10.1007/ s12083-019-00753-z

19. Y. Yuan, W. Liu, T. Wang, Q. Deng, A. Liu, H. Song. Compressive sensing based clustering joint annular routing data gathering scheme for wireless sensor networks. IEEE Access, (2019) Dol: https://doi.org/10. 1109/ACCESS.2019.2935462

20. C. Lin, YX He, N Xiong. An energy-efficient dynamic power management in wireless sensor networks. Fifth International Symposium on Parallel and Distributed Computing, 37, (2006).

21. F. Wang, W. Liu, T. Wang, M. Zhao, M. Xie, H. Song, X. Li, A. Liu, To reduce delay, energy consumption and collision through optimization duty-cycle and size of forwarding node set in WSNs. IEEE Access 7(1), 55983-56015 (2019)

22. S. Sarang, M. Drieberg, A. Awang, R. Ahmad, A QoS MAC protocol for prioritized data in energy harvesting wireless sensor networks. Comput. Netw. 144, 141-153 (2018)

23. H. Cheng, N. Xiong, LT Yang, YS Jeong. Distributed scheduling algorithms for channel access in TDMA wireless mesh networks. The Journal of Supercomputing, 63(2), 407-430 (2013)

24. L. Yin, J. Gui, Z. Zeng. Improving energy efficiency of multimedia content dissemination by adaptive clustering and D2D multicast. Mobile Information Systems, 2019, 5298508 (2019). DOl: https://doi.org/10.1155/2019/5298508.

25. Y. Bi, G. Han, C. Lin, X. Wang, Q. Zhang, Z. Liu, Effective packet loss elimination in IP mobility support for vehicular networks. IEEE Netw. (2019). https://doi.org/10.1109/MNET.2019.1900093

26. N. Xiong, AV Vasilakos, J Wu, YR Yang, A Rindos, Y Zhou, WZ Song. A selftuning failure detection scheme for cloud computing service. 2012 IEEE 26th International Parallel and Distributed Processing Symposium. 40, (2012).

27. X. Liu, M. Zhao, A. Liu, K. Wong, Adjusting forwarder nodes and duty cycle using packet aggregation routing for Body Sensor Networks. Information Fusion 53, 183-195 (2020)

28. Y. Liu, A. Liu, Y. Li, Z. Li, Y. Choi, H. Sekiya, J. Li, APMD: A fast data transmission protocol with reliability guarantee for pervasive sensing data communication. Pervasive and Mobile Computing 41, 413-435 (2017)

29. Y. Yang, N. Xiong, NY Chong, X Défago. A decentralized and adaptive flocking algorithm for autonomous mobile robots. The 3rd International Conference on Grid and Pervasive Computing, (2008).

30. X. Deng, T. He, L. He, J. Gui, Q. Peng, Performance analysis for IEEE 802.11 s wireless mesh network in smart grid. Wirel. Pers. Commun. 96(1), 1537-1555 (2017)

31. Y. Bi, G. Han, C. Lin, M. Guizani, X. Wang, Mobility Management for intro/ inter domain handover in software defined networks. IEEE Journal on Selected Areas in Communications. 37(8), 1739-1754 (2019)

32. X. Peng, J. Ren, L. She, D. Zhang, J. Li, Y. Zhang, BOAT: A block-streaming app execution scheme for lightweight loT devices. IEEE Internet-of-Things Journal 5(3), 1816-1829 (2018)

33. H. Cheng, D. Feng, X. Shi, et al., Data quality analysis and cleaning strategy for wireless sensor networks. EURASIP J. Wirel. Commun. Netw. 2018, 61 (2018). https://doi.org/10.1186/s13638-018-1069-6

34. Y. Liu, A. Liu, N. Xiong, T. Wang, W. Gui. Content propagation for contentcentric networking from location-based social networks. IEEE Transactions on Systems Man Cybernetics-Systems, (2019). DOl: https://doi.org/10.1109/ TSMC.2019.2898982

35. G. Zhang, T. Wang, G. Wang, A. Liu, W. Jia. Detection of hidden data attacks combined fog computing and trust evaluation method in sensor-cloud system. Concurrency and Computation: Practice and Experience, (2018). DOl: https://doi.org/10.1002/cpe.5109.

36. H. Cheng, Z. Su, N. Xiong, Y. Xiao, Energy-efficient node scheduling algorithms for wireless sensor networks using Markov Random Field model. Inf. Sci. 329, 461-477 (2016)

37. C. Zhang, R. Chen, L. Zhu, A. Liu, Y. Lin, F. Huang. Hierarchical information quadtree: efficient spatial temporal image search for multimedia stream. Multimedia Tools and Applications, (2018). DOl: https://doi.org/10.1007/ s11042-018-6284-y.

38. W. Liu, P. Zhuang, H. Liang, J. Peng, Z. Huang, Distributed economic dispatch in microgrids based on cooperative reinforcement learning. IEEE Transactions on Neural Networks and Learning 29(6), 2192-2203 (2018) 
39. Y. Liu, A. Liu, Z. Chen, Analysis and Improvement of send-and-wait automatic repeat-reQuest protocols for wireless sensor networks. Wirel. Pers. Commun. 81(3), 923-959 (2015)

40. W. Turin, Throughput analysis of the Go-Back-N protocol in fading radio channels. IEEE Journal on Selected Areas in Communications 17(5), 881-887 (1999)

41. E. Weldon, An improved selective-repeat ARQ strategy. IEEE Trans. Commun. 30(3), 480-486 (1982)

42. A. Laouid, A. Dahmani, A. Bounceur, R. Euler, F. Lalem, A. Tari, A distributed multi-path routing algorithm to balance energy consumption in wireless sensor networks. Ad Hoc Netw. 64, 53-64 (2017)

43. Y. Sang, H. Shen, Y. Tan, N. Xiong. Efficient protocols for privacy preserving matching against distributed datasets. International Conference on Information and Communications Security, 210-227, (2006).

44. A. Liu, Z. Zheng, C. Zhang, Z. Chen, et al., Secure and energy-efficient disjoint multipath routing for WSNs. IEEE Trans. Veh. Technol. 61(7), 32553265 (2012)

45. J. Zhang, A. Liu, P. Hu, J Long. A fuzzy-rule-based packet reproduction routing for sensor networks. International Journal of Distributed Sensor Networks, 14(4), (2018). DOI: 1550147718774016.

46. C. Joo, N.B. Shroff, On the delay performance of in-network aggregation in lossy wireless sensor networks. IEEE/ACM Transactions on Networking (TON) 22(2), 662-673 (2014)

47. M. Huang, A. Liu, T. Wang, C. Huang. Green data gathering under delay differentiated services constraint for Internet of Things. Wireless Communications and Mobile Computing, 2018, 9715428 (2018). DOl: https://doi.org/10.1155/2018/9715428.

48. Q. Chen, S.S. Kanhere, M. Hassan, Analysis of per-node traffic load in multihop wireless sensor networks. IEEE transaction on wireless communications 8(2), 958-967 (2009)

49. A.F. Liu, P.H. Zhang, Z.G. Chen, Theoretical analysis of the lifetime and energy hole in cluster based Wireless. Journal of Parallel and Distributed Computing 71(10), 1327-1355 (2011)

50. Z. Rosberg, R.P. Liu, T.L. Dinh, Y.F. Dong, S. Jha, Statistical reliability for energy efficient data transport in wireless sensor networks. Wirel. Netw 16, 1913-1927 (2010)

51. H. Zhou, J. Wu, H. Zhao, S. Tang, C. Chen, J. Chen, Incentive-driven and freshness-aware content dissemination in selfish opportunistic mobile networks. IEEE Transactions on Parallel and Distributed Systems 26(9), 2493 2505 (2015)

52. T. Wang, H. Luo, W. Jia, A. Liu, M Xie. MTES: An intelligent trust evaluation scheme in sensor-cloud enabled industrial Internet of Things, IEEE Transactions on Industrial Informatics, (2019) Dol: https://doi.org/10.1109/TII. 2019.2930286

53. Y. Liu, A. Liu, X. Liu, M. Ma, A trust-based active detection for cyber-physical security in industrial environments. IEEE Transactions on Industrial Informatics (2019). https://doi.org/10.1109/TII.2019.2931394

54. H. Teng, K. Zhang, M. Dong, K. Ota, A. Liu, M. Zhao, T Wang. Adaptive transmission range based topology control scheme for fast and reliable data collection. Wireless Communications and Mobile Computing, 2018, 4172049 (2018). DOI: https://doi.org/10.1155/2018/4172049.

\section{Publisher's Note}

Springer Nature remains neutral with regard to jurisdictional claims in published maps and institutional affiliations.

\section{Submit your manuscript to a SpringerOpen ${ }^{\oplus}$ journal and benefit from:}

- Convenient online submission

- Rigorous peer review

- Open access: articles freely available online

- High visibility within the field

- Retaining the copyright to your article

Submit your next manuscript at $\boldsymbol{\nabla}$ springeropen.com 